\title{
Hydrogen Bonding and Related Properties in Liquid Water: A Car-Parrinello Molecular Dynamics Simulation Study
}

\author{
Elvira Guardia ${ }^{\dagger, *}$, Ioannis Skarmoutsos ${ }^{\dagger, \ddagger}$ and Marco Masia ${ }^{\S}$ \\ ${ }^{+}$Departament de Física i Enginyeria Nuclear, Universitat Politecnica de Catalunya, Campus Nord B4-B5, \\ Barcelona 08034, Spain, email: elvira.guardia@upc.edu \\ ‡ INAC/SPrAM (UMR 5819 UJF, CNRS, CEA), CEA-Grenoble, 17 Rue des Martyrs, 38054 Grenoble, \\ France.e-mail: ioannis.skarmoutsos@cea.fr \\ ${ }^{\S}$ Dipartimento di Chimica e Farmacia, Universita degli Studi di Sassari, Istituto Officina dei Materiali del CNR, \\ UOS SLACS, Via Vienna 2,07100 Sassari, Italy, email: marco.masia@uniss.it \\ Present Address: Institut für Physikalische und Theoretische Chemie, Goethe Universitat Frankfurt Max-von- \\ Laue-Str. 7, D-60438 Frankfurt am Main, Germany
}

\begin{abstract}
The local hydrogen bonding structure and dynamics of liquid water have been investigated using the Car-Parrinello molecular dynamics simulation technique. The radial distribution functions and coordination numbers around water molecules have been found to be strongly dependent on the number of hydrogen bonds formed by each molecule, revealing also the existence of local structural heterogeneities in the structure of the liquid. The results obtained have also revealed the strong effect of the local hydrogen bonding network on the local tetrahedral structure and entropy. The investigation of the dynamics of the local hydrogen bonding network in liquid water has shown that this network is very labile and the hydrogen bonds break and reform very rapidly. Nevertheless, it has been found that the hydrogen bonding states associated with the formation of 4 hydrogen bonds by a water molecule exhibit the largest survival probability and corresponding lifetime. The reorientational motions of water molecules have also been found to be strongly dependent on their initial hydrogen bonding state. Finally the dependence of the librational and vibrational modes of water molecules on the local hydrogen bonding network has been carefully examined, revealing a significant effect upon the libration and bond-stretching peak frequencies. The calculated low frequency peaks come in agreement with previously reported interpretations of the experimental low frequency Raman spectrum of liquid water.
\end{abstract}

Keywords: liquid water, hydrogen bonding, structure, dynamics, Car-Parrinello Molecular Dynamics 


\section{Introduction}

The importance of water as a fundamental compound for the existence of all living organisms on this planet has been well underlined so far, in view of the fact that it is involved in nearly all chemical, biological and geological processes. A detailed understanding of its properties is therefore essential and at the same time demanding due to its complex behaviour ${ }^{1-5}$, often related to the ability of water molecules to form labile hydrogen-bonding (HB) networks.

One of the most essential questions to address for a microscopic understanding of water is to find how the static and dynamic behavior of these HB networks gives rise to its characteristic properties. From a theoretical point of view, the investigation of HB and structural features of water could be achieved by employing multiscale modeling techniques, ranging from classical or ab initio molecular simulation techniques (molecular dynamics (MD) or Monte Carlo) to coarse-graining ones ${ }^{6-15}$. Numerous empirical potentials for classical simulations of water have been developed so far ${ }^{6,10}$, ranging from very simple ones based on atomic partial charges and rigid bonds to other more sophisticated ones that include molecular flexibility and explicit electronic polarization effects. However, in order to provide a more realistic description of phenomena like hydrogen bonding in water, electronic effects associated with the formation and breaking of hydrogen bonds might be taken into account explicitly ${ }^{8,13-15}$. HB is a complex type of interaction ${ }^{16,17}$, with contributions arising from electrostatic interactions between permanent multipoles, polarization, and induction interactions between permanent and induced multipoles, dispersion arising from instantaneous multipole-induced multipoles, charge-transfer-induced covalency, and exchange correlation effects from short-range repulsion due to overlap of the electron distribution. Therefore a detailed description of these interactions in a 
quantum-chemical framework is required in order to elucidate fundamental questions about the nature of hydrogen bonds in water ${ }^{10}$. One of the most efficient methods to incorporate the above effects in MD simulations of condensed phase molecular systems is to combine finite temperature atomistic MD with internuclear forces obtained from electronic structure calculations performed "on the fly" as the simulation proceeds ${ }^{18}$. For reasons of computational efficiency, the most commonly employed approach to represent the electronic structure and corresponding interactions is based on density functional theory (DFT). In particular, the CarParrinello MD (CPMD) scheme ${ }^{19}$ has been successfully employed to study a wide variety of chemically interesting systems including water ${ }^{8,9,11-15}$.

The main aim of this work is to investigate in detail the structure and dynamics of the HB network in liquid water using the CPMD simulation technique. More specifically, the effect of $\mathrm{HB}$ interactions on the local intermolecular structure around the individual water molecules and its impact on their reorientational and translational dynamics has been addressed through a systematic investigation of the impact of HB interactions on the librational and vibrational motions in liquid water. Finally, the interrelation between the local HB network and the tetrahedral structural arrangement around each water molecule has been assessed, providing quantitative descriptions of these phenomena for the first time in the literature. This paper is organized as follows: the computational details are presented in Section II. Results and discussion are presented in Section III. General conclusions are presented in Section IV. 


\section{Simulation Details}

Ab initio MD simulations were performed using the Car- Parrinello (CP) ${ }^{19}$ scheme for propagating the wavefunctions and the ionic configurations as implemented in the CPMD package ${ }^{20}$. The BLYP density functional ${ }^{21,22}$ was used for the electronic structure calculations. The cutoff for the wavefunction was set to $80 \mathrm{Ry}$, the time step was set to 4 a.u. and the fictitious mass for the orbital was chosen to be 400 a.m.u. A cubic simulation box containing 96 water molecules at the density of $0.997 \mathrm{~g} \mathrm{~cm}^{-3}$ was used. Periodic boundary conditions were applied. DFT is known to be problematic at reproducing dispersion interactions. In the last years, new methods have been proposed for including dispersion in DFT calculations (see Ref. 23 for a recent review article). One of these methods is based on the use of dispersioncorrected atom-centered pseudopotentials (DCACPs) ${ }^{24,25}$. With DCACP a dispersion potential is included in the pseudopotential; its main advantage is that the electron density coming out of the electronic structure calculation contains the dispersion contribution and there is no need to add any empirical parameter. The additional artificial potential implemented in DCAC pseudopotentials is optimized to reproduce the reference interaction energy from sophisticated high level quantum chemical calculations. In the present study we have used DCACPs in the Troullier-Martins ${ }^{26}$ format for oxygen and hydrogen. It has been recently shown that these pseudopotentials successfully account for London dispersion forces, and that they are capable of faithfully reproducing many dynamical and structural properties of water ${ }^{25}$. A pre-equilibration of the system was done with classical MD simulations using a flexible water model parametrized from ab initio reference data ${ }^{27}$. Following Lin et al. ${ }^{25}$, production runs of 15 ps in the microcanonical ensemble followed NVT equilibration runs of 3 ps where the temperature was set to $330 \mathrm{~K}$; the initial 
configurations were generated with classical MD simulations of 200 ps. Here we would like to highlight that the reference temperature was set to $330 \mathrm{~K}$ in order to avoid falling into the temperature range where BLYP simulations suffer for nonergodic behavior ${ }^{7}$ on time scales shorter than 20 ps. In order to ensure that the simulations are long enough to calculate reliable autocorrelation functions, classical simulations were performed to compare small systems over short times to large systems on extended time scales: it was found that the finite length and time scale effects on the obtained dynamic properties of water are not so strong due to the fact that the dynamical phenomena in liquid water are fast. A more detailed description of the system size effects on the calculated static and dynamic properties of liquid water can be found in the Supporting Information section.

\section{Results and Discussion}

\section{A. Local Structure - HB Network}

In order to reveal some information relative with the local intermolecular structure in liquid water, the atom-atom pair radial distribution functions (prdf) $\mathrm{O}-\mathrm{H}, \mathrm{O}-\mathrm{O}$ and $\mathrm{H}$ $\mathrm{H}$ were calculated and they are depicted in Fig. 1-top panel. By inspecting Fig 1 the good agreement of our results with the experiments ${ }^{28}$ may be clearly noticed. The existence of strong HB interactions is clearly reflected on the positions of the peaks and minima of the calculated prdfs. The $\mathrm{O}-\mathrm{H}$ prdf starts to exhibit non-zero values at very short distances, close to $1.23 \AA$, and the first peak is located at about $1.83 \AA$.

The first minimum of the $\mathrm{O}-\mathrm{H}$ prdf is located at about $2.48 \AA$, followed by a second peak located at $3.28 \AA$, with higher amplitude than the first one, and a second minimum at $4.53 \AA$. The calculated coordination number corresponding to the first minimum of the $\mathrm{O}-\mathrm{H}$ prdf is 1.92 , in reasonable agreement with the experimental 
value of 1.81 recently reported by Soper and Benmore ${ }^{29}$. The O-O prdf starts exhibiting non-zero values at larger distances than in the case of the $\mathrm{O}-\mathrm{H}$, close to $2.33 \AA$ and its first peak is located at about $2.78 \AA$. This peak position and intensity are also in very good agreement with the experimental values recently reported in the x-ray diffraction study of Skinner et al ${ }^{30}$. The first minimum is located at $3.38 \AA$, followed by a second peak located at $4.48 \AA$ and a second minimum at $5.58 \AA$. Finally, concerning the $\mathrm{H}-\mathrm{H}$ prdf, its first non-zero value is located at $1.53 \AA$ and the first peak is located at $2.33 \AA$. The first minimum is located at $2.93 \AA$, followed by a second small peak at $3.93 \AA$.

In order to have a more quantitative representation of the extent of the degree of HB in liquid water a HB analysis was performed, which was based on simple geometric criteria using the positions of the first minima of the calculated $\mathrm{O}-\mathrm{O}$ and $\mathrm{O}-\mathrm{H}$ prdfs. According to the criterion used in the present study, a hydrogen bond between two water molecules exists if the intermolecular distances are $\mathrm{R}_{\mathrm{O}} \ldots \mathrm{O} \leq 3.38 \AA, \mathrm{R}_{\mathrm{H} \ldots \mathrm{O}} \leq$ $2.48 \AA$ and the angle $\phi_{H-O \ldots O} \leq 30^{\circ}$ (here ... denotes an intermolecular bond vector, whereas - denotes an intramolecular one). At this point it has to be mentioned that very similar geometric criteria have been successfully used in the past to predict the HB properties of water and alcohols ${ }^{9,31-43}$. Using this simple geometric criterion, the calculated average number of hydrogen bonds per water molecule $<\mathrm{n}_{\mathrm{HB}}>$ has been estimated to be 3.48, in agreement with the value of 3.58 obtained by reverse modelling of neutron diffraction data ${ }^{44}$.

The calculations have been extended to incorporate hydrogen-bond statistics, by estimating the percentage distribution $f_{n}$ of molecules with $n(n=0-5)$ bonds per molecule, where $\mathrm{f}_{0}$ denotes the percentage of non-bonded molecules or monomer, $\mathrm{f}_{1}$ the percentage of the molecules with one $\mathrm{H}$-bond etc. The results obtained are 
presented in Fig. 1-bottom panel and Table 1 and reveal that the majority of water molecules form 4 hydrogen bonds, however there are significant fractions of molecules forming 2, 3 and 5 bonds. Also, there is a very small fraction of molecules forming zero and one hydrogen bonds.

To investigate in more details the local intermolecular structure in liquid water, the structure around water molecules in different HB states was studied by calculating the atom-atom prdfs $g^{n}{ }_{i j}(r)$, considering the central i water molecule to be in the nth HB state (forming $\mathrm{n}$ hydrogen bonds, $\mathrm{n}=0 . .5$ ). Therefore the $\mathrm{O}-\mathrm{H}, \mathrm{O}-\mathrm{O}$ and $\mathrm{H}-\mathrm{H}$ prdfs were calculated taking into account the $\mathrm{HB}$ state of the central molecule and the results obtained are depicted in Figs. 3, 2 and 4. From these results it may be clearly observed that the calculated prdfs depend very strongly on the HB state of the central water molecule. Especially the structure around HB-free molecules is significantly different, something that is clearly reflected on the O-H prdf which does not exhibit the characteristic first peak observed at very small intermolecular distances, but only the second peak located at $3.48 \AA$. As the number of hydrogen bonds formed by a water molecule increases, then the corresponding $\mathrm{O}-\mathrm{H}$ prdf starts exhibiting the first peak and the intensity of the peak increases when the molecule is in a higher HB state. Note that in order to investigate the system size effects on the behavior of the calculated O-O rdfs around molecules forming 0-5 $\mathrm{H}$. Bonds, these functions were also calculated from the trajectories of a classical MD study with 256 SPCE water molecules and 200 ps duration (see figure S3 in the Supporting Information section). The main features of the functions remain the same; indicating that the obtained functions for the size and time scale reported in our simulation are statistically reliable. 
Similar conclusions may be drawn when inspecting the behavior of the $\mathrm{O}-\mathrm{O}$ and $\mathrm{H}-\mathrm{H}$ prdfs for water molecules in different HB states. It is interesting to notice the appearance of a second peak in the O-O prdf for the $n=1,2$ HB states which disappears for the higher ones, where only a sharp peak located at smaller intermolecular distances and exhibiting higher amplitudes is observed. The differences in the structure around water molecules in different HB states could be also easily observed by inspecting the shape of the $\mathrm{H}-\mathrm{H}$ prdfs. For water molecules in the lowest HB states $(n=0,1)$ two peaks with small and similar amplitudes may be observed, indicating the existence of weak $\mathrm{H}-\mathrm{H}$ interactions. At higher HB states the first peak is much more pronounced than the second one and its amplitude increases as the number of hydrogen bonds formed by the central molecule increases. Moreover it is shifted towards smaller intermolecular distances, at about $2.33 \AA$, whereas in lower HB states its located at larger distances (3.13 and $2.83 \AA$ for $n=0,1$ respectively).

The structure around water molecules which are in different HB states was also investigated by calculating the corresponding coordination numbers as a function of the distance around these molecules. These coordination numbers can be estimated from the calculated $g^{n}{ }_{i j}(r)$ functions using the relation:

$$
N^{n}{ }_{C}(r)=4 \pi \rho \int_{0}^{r} g^{n}{ }_{i j}(R) \cdot R^{2} \cdot d R
$$

In this equation $\rho$ is the bulk density of water. The O-O coordination numbers for some representative HB states of water molecules are presented in Fig. 5. At small intermolecular distances, the first shell of water molecules at higher HB states (eg forming 5 or 3 hydrogen bonds) is over coordinated in comparison with HB free molecules, something which is clearly reflected on the behavior of $N^{n}{ }_{C}(r)$ up to 3.5 
$\AA$. However, this behavior changes for the second solvation shell, where the concentration of neighbor water molecules is slightly higher in the case of HB free molecules than for water molecules at higher HB states. Such an observation indicates the existence of local spatial heterogeneities in the structure of liquid water, which are affected by the HB state of the water molecules in the system.

\section{B. Tetrahedral Structure and Entropy}

A measure of the local structural order in water can be provided by the tetrahedral order parameter $\mathrm{q}^{45}$. This parameter gives information about the extent to which a molecule and its four nearest neighbors adopt a tetrahedral arrangement and is defined as:

$$
q=1-\left\langle\frac{3}{8} \sum_{j=1}^{3} \sum_{k=j+1}^{4}\left(\cos \phi_{j i k}+\frac{1}{3}\right)^{2}\right\rangle
$$

In this equation $\phi_{j i k}$ corresponds to the angle formed by the vectors $\vec{r}_{i j}$ and $\vec{r}_{i k}$, connecting the oxygen atom of the central molecule i with the oxygen atoms of two of its four nearest neighbours $\mathrm{j}$, $\mathrm{k}$. Using this definition the parameter q gets the value $\mathrm{q}=1$ in a perfect tetrahedral network and the value $\mathrm{q}=0$ in an ideal gas ${ }^{45}$. In the present study the average orientational tetrahedral order parameter $\mathrm{q}$ and the normalized probability density distribution function $\mathrm{f}(\mathrm{q})$ were calculated.

$\mathrm{f}(\mathrm{q})$ for liquid water is presented in Fig. 6-top panel and the average value of q has been estimated to be $\langle\mathrm{q}>=0.678$. Interestingly, the value of the orientational tetrahedral order parameter calculated in the presented study is very similar with the values reported in recent DFT-based ab initio MD studies using hybrid exchange functionals to account for exact exchange and taking into account non-local Van der Waals/dispersion interactions, as well as nuclear quantum effects ${ }^{46}$. These values are 
lower in comparison with the values obtained when using a PBE-GGA level of theory and much closer to the value of 0.58 , which was obtained by the reverse modelling of neutron diffraction data ${ }^{29}$. Recent studies by Zhang et $\mathrm{al}^{12}$ have also pointed out that although the PBE-GGA level of theory predicts quite accurately the total entropy of liquid water, having a 1-2\% difference with the experimental data, it overestimates the energy difference between hydrogen bonded and non-hydrogen bonded configurations and thus the liquid turns out to be more ordered.

To investigate also the effect of the HB state of water molecules in the value of the orientational tetrahedral order parameter, the values of $<\mathrm{q}>$ for water molecules forming $n_{\mathrm{HB}}=0-5$ hydrogen bonds have been calculated separately and are presented as a function of $n_{\mathrm{HB}}$ in Fig. 6-bottom panel). The value of $<\mathrm{q}>$ increases linearly with the increase of $n_{H B}$ and exhibits its maximum value $<\mathrm{q}>=0.756$ for water molecules forming four hydrogen bonds. For water molecules forming five hydrogen bonds the value of $<\mathrm{q}>$ decreases to 0.685 . All these observations clearly signify that the formation of hydrogen bonds is the most important factor affecting the tetrahedral structural arrangements in water and this specific local structure is mostly favored when a water molecule forms four hydrogen bonds with its neighbors.

The dynamic behavior of the orientational tetrahedral order parameter has also been investigated, by calculating the corresponding average time correlation function (tcf):

$$
C_{q}(t)=\frac{\left\langle\delta q_{i}(0) \cdot \delta q_{i}(t)\right\rangle}{\left\langle\delta q_{i}(0)^{2}\right\rangle}, \delta q_{i}(t)=q_{i}(t)-\langle q\rangle
$$

The corresponding correlation time $\tau_{q}$ associated with the relaxation of this orientational tetrahedral order parameter has been calculated using the relation:

$$
\tau_{q}=\int_{0}^{\infty} C_{q}(t) \cdot d t
$$


The calculated tcf $C_{q}(t)$ decays very rapidly for the first $0.2 \mathrm{ps}$ and then decays much slower until it reaches the zero value at about 3.5 ps (see the Supporting Information Section). A double exponential decay function has been fitted to the calculated one and the corresponding correlation time $\tau_{q}$ has been estimated to be 0.40 ps.

In recent studies the local tetrahedral order has been correlated to the entropy of the system $^{12,47}$; by considering that the order parameter of each molecule are independent, and provided the distribution of states q is continuous, the "tetrahedral entropy" per molecule (see Supporting Information for more details) is defined as:

$$
S_{q}-S_{0}=\frac{S\left(q_{1}, q_{2}, \ldots, q_{N}\right)}{N}-S_{0}=\frac{3}{2} \cdot K_{B} \int \ln (1-q) \cdot f(q) \cdot d q
$$

where $\mathrm{S}_{0}$ is a constant solely depending on the simulation cell volume. Given the additive property of the integral, the contributions of different classes of H-bonded molecules could be easily isolated. Being $\chi_{i}=\left\langle n_{i}\right\rangle / N$ the fraction of molecules of class i (i ranging from 0 to 5), the total entropy per molecule is given by $S_{q}=S_{0}+\sum_{n=0}^{5} \chi_{n} \cdot S_{q}{ }^{n}$, where the contribution to the entropy of each class is:

$$
S_{q}{ }^{n}=\frac{3}{2} \cdot K_{B} \int_{q_{\min }}^{q_{\max }} \ln (1-q) \cdot f^{n}(q) \cdot d q
$$

where the integration is performed over the field of existence of the function $f^{n}(q)$. In figure 7 the distributions $f^{n}(q)$ are shown; they are rather broad for low values of $\mathrm{n}$ because non-hydrogen bonded molecules around the central water molecule reorient easier (see below) and are less constrained to a rigid structure. On the other hand, $f^{n}(q)$ class peak at values closer to 1 for molecules with more than 3 HBs. Particularly, when $4 \mathrm{HBs}$ are formed it is more likely that the tetrahedral order is higher because the 4 molecules are easily accommodated in such structure. Hence, the 
tetrahedral entropy term associated to molecules forming four HBs (Eq. 6) is the lowest one; this can be seen in figure 8. Given that the fraction of molecules forming four HBs is the highest one (figure 1), their contribution to the overall tetrahedral entropy becomes significantly important and lowers the average value per molecule.

\section{Hydrogen Bond Dynamics}

The average HB dynamics for pairs i, j of hydrogen bonded molecules could be described using the following time correlation function ${ }^{31,34,35,48-51}$ :

$$
C_{H B}(t)=\frac{\left\langle h_{i j}(0) \cdot h_{i j}(t)\right\rangle_{t^{*}}}{\left\langle h_{i j}(0)^{2}\right\rangle}
$$

The corresponding HB lifetime is defined as:

$$
\tau_{H B}=\int_{0}^{\infty} C_{H B}(t) \cdot d t
$$

The variable $h_{i j}(t)$ has been defined in the following way:

$h_{i j}(t)=1$, if molecule $\mathrm{j}$ is hydrogen bonded with molecule $\mathrm{i}$ at times 0 and $\mathrm{t}$ and the bond has not been broken in the meantime for a period longer than $t^{*} ; h_{i j}(t)=0$, otherwise.

Using this definition, the calculation of $C_{H B}(t)$ depends upon the selection of the parameter $t^{*}$. The two limiting cases arising from this definition are:

1. If $t^{*}=0$ which represents the so-called continuous definition. In this case the calculated tcf is the continuous one, $C_{H B}{ }^{C}(t)$, and the corresponding lifetime is the continuous lifetime $\tau_{H B}{ }^{C}$. 
2. If $t^{*}=\infty$ which represents the so-called intermittent definition. In this case the calculated tcf is the intermittent one, $C_{H B}{ }^{I}(t)$, and the corresponding lifetime is the intermittent lifetime (or HB relaxation time) $\tau_{H B}{ }^{I}$.

These two definitions describe very different aspects of HB dynamics and have been well described in previous publications ${ }^{31,36}$. The corresponding lifetimes $\tau_{H B}{ }^{C}$ and $\tau_{H B}{ }^{I}$ have been estimated to be 0.46 and 4.9 ps, respectively. These values are in good agreement with the values obtained using classical force fields ${ }^{31}$ which predict a wide range of water properties at different thermodynamic state points.

The dynamics of the HB network in liquid water were further investigated, taking into account the dynamics of the different $\mathrm{HB}$ states of the water molecules and calculating the appropriate tcfs. The intermittent HB state dynamics have been investigated by calculating the function ${ }^{41,43}$ :

$$
C_{H B}^{n, I}(t)=\frac{\left\langle\delta h_{n}{ }^{I}(0) \cdot \delta h_{n}{ }^{I}(t)\right\rangle}{\left\langle\delta h_{n}{ }^{I}(0)^{2}\right\rangle}, \delta h_{n}{ }^{I}(t)=h_{n}{ }^{I}(t)-\left\langle h_{n}{ }^{I}\right\rangle
$$

The variable $h_{n}{ }^{I}(t)$ has been defined in the following way:

$h_{n}{ }^{I}(t)=1$ if a water molecule that was in the $\mathrm{n}^{\text {th }} \mathrm{HB}$ state at time $\mathrm{t}=0$ is in the same HB state at time t, independently of whether or not its HB state has changed in the meantime; $h_{n}{ }^{I}(t)=0$, otherwise. The corresponding lifetimes have been defined as:

$$
\tau_{H B}^{n, I}=\int_{0}^{\infty} C_{H B}^{n, I}(t) \cdot d t
$$

These functions can provide information about the survival probability of HB states overlooking the very fast processes of forming and breaking single hydrogen bonds. The intermittent HB state tcfs for $\mathrm{n}=0-5$ are depicted in Fig. 9. The corresponding lifetimes are presented in Table 1 . The slowest decay has been observed for the 
$C_{H B}^{4, I}(t)$ tcf, signifying that the survival probability of the $\mathrm{n}=4 \mathrm{HB}$ state of water molecules forming initially 4 hydrogen bonds decays much slower than for the other HB states, especially the $n=0,1$ HB states where the calculated tcfs decay very fast. The calculated lifetimes $\tau_{H B}^{n, I}$ have also been found to be significantly lower than those corresponding to alcohols ${ }^{41}$. Such an observation indicates that although the average number of hydrogen bonds per water molecule is much higher than the corresponding ones for alcohols, the HB network in water is much more labile. Therefore, hydrogen bonds in water can break and reform much faster changing thus more rapidly the HB state of the water molecules.

\section{Reorientational Dynamics}

Reorientation of water significantly affects important microscopic phenomena like proton transfer and transport ${ }^{52-55}$ and protein hydration ${ }^{56}$ and it is closely related to the dynamics of the hydrogen bond network. For this reason several experimental ${ }^{57-64}$, and theoretical studies ${ }^{65-69}$ have been devoted to the investigation of molecular reorientation in liquid water and its relationship with the formation and breaking of hydrogen bonds. The most widely used mechanism to describe molecular reorientation up to now was the Debye small-step diffusion model ${ }^{38}$. However, recent classical MD simulation studies ${ }^{66-68}$ have revealed that water reorientation takes place through large angular jumps, involving the breaking of a hydrogen bond between the rotating molecule and an overcoordinated first-shell neighbor and the creation of another hydrogen bond with an undercoordinated second-shell neighbor.

Taking into account these observations, the reorientational dynamics of water molecules in different initial HB states were investigated in order to reveal the effect 
of the HB state on these dynamics. Initially, the average Legendre reorientational tcfs for the water molecules were calculated, using the relation:

$$
C_{\ell R}(t)=\frac{\left\langle P_{\ell}(\vec{u}(0) \cdot \vec{u}(t))\right\rangle}{\left\langle P_{\ell}(\vec{u}(0) \cdot \vec{u}(0))\right\rangle}, \quad \ell=1-3
$$

In this equation $\vec{u}$ is a unit vector along a specified direction inside a molecule and $P_{\ell}$ is a Legendre polynomial $\left(P_{1}(x)=x, P_{2}(x)=\frac{1}{2}\left(3 x^{2}-1\right), P_{3}(x)=\frac{1}{2}\left(5 x^{3}-3 x\right)\right)$. The index $\ell$ defines the order of the Legendre polynomial. The corresponding reorientational times are defined as:

$$
\tau_{\ell R}=\int_{0}^{\infty} C_{\ell R}(t) \cdot d t \quad, \ell=1-3
$$

The calculated reorientational times for the $\mathrm{O}-\mathrm{H}$ vector are $\tau_{1 R}=4.8 \mathrm{ps}, \tau_{2 R}=2.0 \mathrm{ps}$ and $\tau_{3 R}=1.0 \mathrm{ps}$. According to the Debye diffusive model the ratio of these values has to be $\tau_{1 R} / \tau_{2 R}=3$ and $\tau_{1 R} / \tau_{3 R}=6^{68}$, something which obviously is not the case for the calculated $\tau_{1 R}, \tau_{2 R}$ and $\tau_{3 R}$ values of water in the framework of the present study. Such an observation clearly indicates that the small-step Debye diffusion model is not very adequate in describing the reorientational motions in liquid water, as it was pointed out in recent classical MD studies as well ${ }^{66-68}$. The most typical characteristic of Legendre tcfs is the presence of a minimum at very short time scales (0.03ps) which is more pronounced as the order of the Legendre polynomial increases. Such a minimum has been attributed to the reorientational motions related to the breaking and creation of hydrogen bonds; being due to librations, the delay reflects the strength of the hydrogen-bond ${ }^{67,70}$.

In order to investigate the effect of the HB state of the individual water molecules on their O-H reorientational dynamics the Legendre reorientational tcfs as a function of 
the initial HB state of these molecules were calculated. These functions can be defined as follows ${ }^{36}$ :

$$
C_{n, \ell R}(t)=\frac{\left\langle P_{\ell}(\vec{u}(0) \cdot \vec{u}(t)) \cdot \theta_{n}(0)\right\rangle}{\left\langle P_{\ell}(\vec{u}(0) \cdot \vec{u}(0)) \cdot \theta_{n}(0)\right\rangle}, n=0-5, \ell=1-3
$$

The index $\mathrm{n}$ defines the instantaneous number of hydrogen bonds which a molecule forms at each time t. The function $\theta_{n}(t)$ is defined as follows: $\theta_{n}(t)=1$ if a molecule forms $n$ hydrogen bonds at time $\mathrm{t} ; \theta_{n}(t)=0$, otherwise.

The calculated tcfs $C_{n, \ell R}(t)(\mathrm{n}=0-5)$ are presented in Fig. 10. The short time decay of these functions is very sensitive on their initial HB state and water molecules initially forming 4 or 5 hydrogen bonds reorient much slower than molecules initially found at lower HB states. This is more clearly reflected on the behavior of the second and third order Legendre tcfs, which decay much more rapidly in the cases of molecules initially being at the lowest HB states. These functions exhibit a minimum at a very short time period, close to $0.03-0.05$ ps. The location of this minimum is observed at shorter time scales for higher HB states. Afterwards, the values of these tcfs increase for a very short time period of 0.01-0.02 ps and when they reach a local maximum they start decaying much slower. This slower time decay is very similar for all the reorientational tcfs $C_{n, \ell R}(t)$. Therefore the decay of the calculated reorientational tcfs at larger time scales ( $\mathrm{t}>0.1 \mathrm{ps}$ ) resembles a diffusive behavior and can be described by a single exponential decay function. Interestingly, the calculated reorientational tcfs exhibit their minimum at the same time when the intermittent HB state tcfs also exhibit a similar shoulder (see Fig. 10). The time decay of the intermittent HB state tcfs after this time period also becomes significantly slower. Such an observation clearly indicates that the reorientation of water molecules is closely related to the 
changes of their HB state and these changes are significantly affecting the short time behavior of the reorientational tcfs.

\section{E. Atomic Translational Dynamics - Spectral Densities}

In the present treatment the effect of the local HB structure around the individual water molecules on their single translational dynamics was also investigated. To do so, the hydrogen and oxygen atomic velocity tcfs were calculated using the relation:

$$
C_{v}{ }^{j}(t)=\frac{\left\langle\vec{v}_{j}(0) \cdot \vec{v}_{j}(t)\right\rangle}{\left\langle\vec{v}_{j}(0)^{2}\right\rangle}, j \equiv H, O
$$

The corresponding spectral densities $S_{v}{ }^{j}(\omega)$ have also been calculated by performing a cosine transform on the tcfs:

$$
S_{v}{ }^{j}(\omega)=\int_{0}^{\infty} \cos (\omega \cdot t) \cdot C_{v}{ }^{j}(t) \cdot d t, j \equiv H, O
$$

The spectral densities have been calculated by numerical integration using a Bode rule, after applying a Hanning window to the calculated atomic velocity tcfs. The calculated $S_{v}{ }^{H}(\omega)$ spectral density exhibits three characteristic peaks at 523, 1584 and $3308 \mathrm{~cm}^{-1}$ which correspond to the librational, bending and stretching motions in water $^{31,71-73}$. A very weak low-frequency band located at $61 \mathrm{~cm}^{-1}$ has also been observed. In the case of $S_{v}{ }^{O}(\omega)$, a characteristic peak at $56 \mathrm{~cm}^{-1}$ and a shoulder centered about $175 \mathrm{~cm}^{-1}$ may be observed. The peak and shoulder positions of the calculated oxygen spectral density $S_{v}{ }^{o}(\omega)$ are very close to the experimentally observed weak bands at 60 and $170 \mathrm{~cm}^{-1}$ in the low-frequency region of the Raman spectrum of liquid water ${ }^{71,74,75}$. 
In order to investigate the effect of the initial HB state of water molecules on the positions of the vibrational and librational bands in liquid water, the following tcfs were calculated $^{36}$ :

$$
C_{n, v}{ }^{j}(t)=\frac{\left\langle\vec{v}_{j}(0) \cdot \vec{v}_{j}(t) \cdot \theta_{n}(0)\right\rangle}{\left\langle\vec{v}_{j}(0)^{2} \cdot \theta_{n}(0)\right\rangle}, n=0-5, j \equiv H, O
$$

The corresponding spectral densities were then obtained by the cosine transform of these tcfs:

$$
S_{n, v}^{j}(\omega)=\int_{0}^{\infty} \cos (\omega \cdot t) \cdot C_{n, v}^{j}(t) \cdot d t, n=0-5, j \equiv H, O
$$

The calculated hydrogen and oxygen spectral densities for different initial HB states of water molecules are depicted in Figs. 11 and 12, respectively. By inspecting Fig. 11, it may be observed that the libration and bond-stretching peaks of water molecules are affected by their initial HB state, whereas the angle bending frequencies are independent of the HB state of water molecules. The libration, angle bending and bond stretching peak positions corresponding to the several initial HB states of water molecules are presented in Table 2. As the number of hydrogen bonds initially formed by a water molecule increases the bond-stretching peak positions are red-shifted, whereas the libration peak positions are blue-shifted. Going from HB-free water molecules to molecules forming 5 hydrogen bonds the red-shift in the stretching peak position has been estimated to be about $160 \mathrm{~cm}^{-1}$, whereas for the libration peak positions the corresponding blue-shift is about $200 \mathrm{~cm}^{-1}$. Interestingly, in a recent CarParrinello study of liquid $\mathrm{D}_{2} \mathrm{O}^{76}$ it has been also pointed out that the lower O-D vibrational frequency modes primarily involve strongly hydrogen bonded OD modes. The O-D stretching frequency for the isolated, hydrogen bonded free, gas water molecule has been estimated to be $2560 \mathrm{~cm}^{-1}$ and in liquid water it has been found to 
be $2380 \mathrm{~cm}^{-1}$. This red-shift by $180 \mathrm{~cm}^{-1}$ is in absolute agreement with our results concerning the red-shift in the $\mathrm{O}-\mathrm{H}$ stretching frequency when going from hydrogen bonded free water molecules to strongly hydrogen bonded ones.

Moreover, the low frequency peak observed in the average $S_{v}{ }^{H}(\omega)$ is not present for initially HB-free molecules. For the oxygen spectral densities $S_{n, v}{ }^{o}(\omega)$, the low frequency peak is also absent for initially HB-free molecules $(n=0)$, however it appears for the higher HB states; exhibiting similar peak positions (Fig. 12). According to the literature ${ }^{77-83}$, the designation for the low frequency band in the Raman spectrum of liquid water observed experimentally at about $60 \mathrm{~cm}^{-1}$ is still a subject of debate; however it is now accepted that the presence of this band is due to a mixture of underlying mechanisms, including hydrogen bridge bonds and cage effects. The shoulder observed at higher frequencies for the average spectral density $S_{v}{ }^{O}(\omega)$, starts to become more apparent at the higher HB states, exhibiting a peak at $175 \mathrm{~cm}^{-1}$ for water molecules forming 5 hydrogen bonds. This finding that such a behavior is more pronounced for strongly hydrogen bonded water molecules is consistent with the interpretation of this band as very closely related to $\mathrm{HB}$ interactions in liquid water. According to previously reported experimental studies ${ }^{77}$, the existence of the $170 \mathrm{~cm}^{-1}$ band in the low frequency Raman spectrum of liquid water has been attributed to the intermolecular stretching vibrational modes of hydrogen bonded water dimers. In the recent Car-Parrinello study of Mallik et al. ${ }^{76}$, the calculation of the power spectra of the relative velocity of an initially hydrogen bonded O...O pair has also revealed a peak at $180 \mathrm{~cm}^{-1}$, further confirming these statements. Therefore, the findings of the present study come in agreement with the previously reported interpretations of the experimental Raman spectroscopic studies in liquid water. 


\section{Conclusions}

In the present treatment, a Car-Parrinello molecular dynamics simulation of liquid water has been performed in order to reveal detailed information about the effect of HB interactions on the local structure and dynamics in the system. The average number of hydrogen bonds per molecule has been estimated to be about 3.5 and the corresponding hydrogen bond statistics have revealed the fraction of water molecules forming 0-5 hydrogen bonds. The results obtained have revealed that the majority of water molecules form 4 hydrogen bonds, however there are significant fractions of molecules forming 2,3 and 5 bonds. Also, there is a very small fraction of molecules forming zero and one hydrogen bonds.

The local structure around water molecules forming 0-5 hydrogen bonds has been investigated in terms of the atom-atom $\mathrm{O}-\mathrm{H}, \mathrm{O}-\mathrm{O}$ and $\mathrm{H}-\mathrm{H}$ intermolecular prdfs and corresponding coordination numbers. The calculated prdfs exhibit significant differences as the number of hydrogen bonds formed by the central water molecule changes, signifying the strong effect of HB interactions on the local structure of the system. At small intermolecular distances, the first shell of water molecules at higher HB states is overcoordinated in comparison with HB free molecules, something which is clearly reflected on the behavior of $N^{n}{ }_{C}(r)$ up to $3.5 \AA$. However, the fact that in the second solvation shell the concentration of neighbor water molecules is slightly higher for water molecules at the lowest HB states, indicates the existence of local spatial heterogeneities in the structure of liquid water. The orientational tetrahedral order parameter q has also been estimated as a function of the hydrogen bonds formed by each central water molecule. The results obtained have shown a very strong dependence of $<\mathrm{q}>$ on the hydrogen bonds formed by each water molecule and the 
maximum value of $<\mathrm{q}>$ is observed around water molecules forming 4 hydrogen bonds, which corresponds to the lowest contribution to the tetrahedral entropy.

The dynamics of the single hydrogen bonds formed in the liquid, as well as the dynamics of the different HB states of water molecules have been investigated using the appropriate tcfs. The results obtained have shown that that the survival probability of the HB state of water molecules forming initially 4 hydrogen bonds decays much slower than for the other HB states. The calculated lifetimes $\tau_{H B}^{n, I}$ have also been found to be significantly lower than those corresponding to alcohols. Such an observation indicates that although the average number of hydrogen bonds per water molecule is much higher than the corresponding ones for alcohols, the HB network in water is much more labile. Molecular reorientation in liquid water has been also investigated using the appropriate tcfs and the results obtained indicate that the smallstep Debye diffusion model is not very adequate in describing the reorientational motions in liquid water, as it was pointed out in recent classical MD studies as well. The results obtained reveal that the short time decay of the reorientational tcfs is very sensitive on their initial HB state. It has been found that water molecules initially forming 4 or 5 hydrogen bonds reorient much slower than molecules initially found at lower HB states. This is more clearly reflected on the behavior of the second and third order Legendre tcfs, which decay much more rapidly in the cases of molecules initially being at the lowest HB states. The decay of the calculated reorientational tcfs at larger time scales ( $\mathrm{t}>0.1 \mathrm{ps}$ ) resembles a diffusive behavior and can be described by a single exponential decay function . Finally, the spectral densities of the atomic velocity tcfs were calculated and it has been found that the libration and bondstretching peaks of water molecules are affected by their initial HB state, whereas the 
angle bending frequencies are almost independent of the HB state of water molecules. As the number of hydrogen bonds initially formed by a water molecule increases the bond-stretching peak positions are red-shifted, whereas the libration peak positions are blue-shifted. The calculated low frequency peaks and shoulders at about 60 and $175 \mathrm{~cm}^{-1}$, and the effect of the HB state of the water molecules upon them comes also in agreement with previously reported interpretations of the experimental low frequency Raman spectrum of liquid water.

\section{Acknowledgements}

The authors thankfully acknowledge the computer resources, technical expertise and assistance provided by the Barcelona Supercomputing Center - Centro Nacional de Supercomputación for the projects QCM-2009-1-0014, QCM-2008-3-0012 and QCM-2008-2-0010. The research institution INSTM is also acknowledged by M.M., who is also thankful for the resources given by the Cybersar Project managed by the “Consorzio COSMOLAB”. E.G. acknowledges financial support from the Direcció General de Recerca de la Generalitat de Catalunya (Grant 2009SGR- 1003) and from the Ministerio de Ministerio de Economía y Cometitividad (MINECO) of Spain (Grant FIS2012-39443-C02-01). I.S. acknowledges the HPC-EUROPA 2 project (project n. 228398) for the support of the European Commision - Capacities Area Research Infrastructures, which allowed him to visit the group of M.M. in the University of Sassari and carry out a part of the analysis presented in this work.

\section{Supporting Information Available:}

System size and time-scale effects in the calculated structural and dynamic properties of liquid water obtained by classical simulations, together with a detailed definition of 
the tetrahedral entropy and the dynamics of the tetrahedral order parameter obtained by the quantum Car-Parrinello simulation, are provided in Figures S1-S10 of the Supporting Information.

\section{References}

1) Nilsson, A.; Pettersson, L. G. M. Perspective on the Structure of Liquid Water. Chem. Phys. 2011, 389, 1-34.

2) Wernet, P.; Nordlund, D.; Bergmann, U.; Cavalleri, M.; Odelius, M.; Ogasawara, H.; Naslund, L. A.; Hirsch, T. K.; Ojamae, L.; Glatzel, P. et al. The Structure of the First Coordination Shell in Liquid Water. Science 2004, 304, 995-999.

3) Huang, C. ; Wikfeldt, K. T.; Tokushima, T.; Nordlund, D.; Harada, Y.; Bergmann, U.; Niebuhr, M.; Weiss, T.M.; Horikawa, Y.; Leetmaa, M. et al. The Inhomogeneous Structure of Water at Ambient Conditions. Proc. Natl. Acad. Sci. (USA) 2009, 106, 15214-15218.

4) Soper, A. K. Water: Two Liquids Divided by a Common Hydrogen Bond. J. Phys. Chem. B 2011, 115, 14014-14022.

5) Soper, A. K. Recent Water Myths. Pure Appl. Chem. 2010, 82, 1855-1867.

6) Guillot, B. A Rappraisal of What We Have Learnt During Three Decades of Computer Simulations of Water. J. Mol. Liq. 2002, 101, 219-260.

7) Kuo, I. -F. W.; Mundy, C. J.; McGrath, M. J.; Siepmann, J. I.; VandeVondele, J.; Sprik, M.; Hutter, J.; Chen, B.; Klein, M. L.; Mohamed, F. et al. Liquid Water from First Principles: Investigation of Different Sampling Approaches. J. Phys. Chem. B 2004, 108, 12990-12998.

8) Sharma, M.; Resta, R.; Car, R. Intermolecular Dynamical Charge Fluctuations in Water: A Signature of the H-Bond Network. Phys. Rev. Lett. 2005, 95, 187401. 
9) Kuhne, T. D.; Krack, M.; Parrinello, M. Static and Dynamical Properties of Liquid Water from Principles by a Novel Car-Parrinello-like Approach. J. Chem. Theory. Comput. 2009, 5, 235-241.

10) Paesani, F.; Voth, G. A. The Properties of Water: Insights from Quantum Simulations. J. Phys. Chem. B 2009, 113, 5702-5719.

11) Zhang, C.; Wu, J.; Galli, G.; Gygi, F. Structural and Vibrational Properties of Liquid Water form van der Waals Density Functionals. J. Chem. Theory Comput. 2011, 7, 3054-3061.

12) Zhang, C.; Spanu, L.; Galli, G. Entropy of Liquid Water from Ab Initio Molecular Dynamics. J. Phys. Chem. B 2011, 115, 14190-14195.

13) Møgelhøj, A.; Kelkkanen, A. K.; Wikfeldt, K. T.; Schiøtz, J.; Mortensen, J. J.; Pettersson, L. G. M.; Lundqvist, B. I.; Jacobsen, K. W.; Nilsson, A.; Nørskov, J. K. Ab Initio van der Waals Interactions in Simulations of Water Alter Structure from Mainly Tetrahedral to High-Density-Like. J. Phys. Chem. B 2011, 115, 14149-14160. 14) Lin, I. -C.; Seitsonen, A. P.; Tavernelli, I.; Rothlisberger, U. Structure and Dynamics of Liquid Water from Ab Initio Molecular Dynamics - Comparison of BLYP, PBE and revPBE Density Functionals with and without van der Waals Corrections. J. Chem. Theory. Comput. 2012, 8, 3902-3910.

15) Heyden, M.; Sun, J.; Forbert, H.; Mathias, G.; Havenith, M.; Marx, D. Understanding the Origins of Dipolar Couplings and Correlated Motion in the Vibrational Spectrum of Water. J. Phys. Chem. Lett. 2012, 3, 2135-2140.

16) Arunan, E.; Desiraju, G. R.; Klein, R. A.; Sadlej, J.; Scheiner, S.; Alkorta, I.; Clary, D. C.; Crabtree, R. H.; Dannenberg, J. J.; Hobza, P. et al. Defining the Hydrogen Bond: An Account. Pure Appl. Chem. 2011, 83, 1619-1636. 
17) Rao, F.; Garrett-Roe, S.; Hamm, P. Structural Inhomogeneity of Water by Complex Network Analysis. J. Phys. Chem. B 2011, 114, 15598-15604.

18) Tuckermann, M. E. Ab Initio Molecular Dynamics and Ab Initio Path Integrals. In "Quantum Simulations of Complex Many-Body Systems: From Theory to Algorithms”, eds J. Grotendorst, D. Marx, A. Muramatsu, NIC Series, 2002.

19) Car, R.; Parrinello, M. Unified Approach for Molecular Dynamics and DensityFunctional Theory. Phys. Rev. Lett. 1985, 55, 2471-2474.

20) Copyright IBM Corp. 1990-2006, computer code CPMD version 3.11, (MPI für Festköorperforschung Stuttgart 1997-2001).

21) Becke, A. D. Density-Functional Exchange-Energy Approximation with Correct Asymptotic Behavior. Phys. Rev. A 1988, 38, 3098-3100.

22) Lee, C.; Yang, W.; Parr, R. G. Development of the Colle-Salvetti CorrelationEnergy Formula into a Functional of the Electron Density. Phys. Rev. B 1988, 37, 785-789.

23) Riley, K. E.; Pitoňák, M.; Jurečka, P.; Hobza, P. Stabilization and Structure Calculations for Noncovalent Interactions in Extended Molecular Systems Based on Wave Function and Density Functional Theories. Chem. Rev. 2010, 110, 5023-5063.

24) von Lilienfeld, O. A.; Tavernelli, I.; Rothlisberger, U.; Sebastiani, D. Optimization of Effective Atom Centered Potentials for London Dispersion Forces in Density Functional Theory. Phys. Rev. Lett. 2004, 93, 153004.

25) Lin, I. -C.; Seitsonen, A. P.; Coutinho-Neto, M. D.; Tavernelli, I.; Rothlisberger, U. Importance of van der Waals Interactions in Liquid Water. J. Phys. Chem. B 2009, $113,1127-1131$.

26) Troullier, N.; Martins, J. L. Efficient Pseudopotentials for Plane-Wave Calculations. Phys. Rev. B 1991, 43, 1993-2006. 
27) Akin-Ojo, O.; Wang, F. Developing Ab Initio Quality Force Fields from Condensed Phase Quantum-Mechanics/Molecular-Mechanics Calculations through the Adaptive Force Matching Method. J. Chem. Phys. 2008, 129, 064108.

28) Soper, A. The Radial Distribution Functions of Water and Ice from 220 to $673 \mathrm{~K}$ and at Pressures up to $400 \mathrm{MPa}$. Chem. Phys. 2000, 258, 121-137.

29) Soper, A. K.; Benmore, C. J. Quantum Differences between Heavy and Light Water. Phys. Rev. Lett. 2008, 101, 065502.

30) Skinner, L. B.; Huang, C.; Schlesinger, D.; Pettersson, L. G. M.; Nilsson, A.; Benmore, C. J. Benchmark Oxygen-Oxygen Pair-Distribution Function of Ambient Water from X-Ray Diffraction Measurements with a Wide Q-Range. J. Chem. Phys. 2013, 138, 074506.

31) Marti, J.; Padro, J. A.; Guardia, E. Molecular Dynamics Simulation of Liquid Water Along the Coexistence Curve: Hydrogen Bonds and Vibrational Spectra. J. Chem. Phys. 1996, 105, 639-649.

32) Nieto-Draghi, C.; Bonet-Avalos, J.; Rousseau, B. Dynamical and Structural Behavior of Different Rigid Nonopolarizable Models of Water. J. Chem. Phys. 2003, 118, 7954-7964.

33) Marti, J. Dynamic Properties of Hydrogen-Bonded Networks in Supercritical Water. Phys. Rev. E 2000, 61, 449-456.

34) Luzar, A.; Chandler, D. Effect of Environment on Hydrogen Bond Dynamics in Liquid Water. Phys. Rev. Lett. 1996, 76, 928-931.

35) Luzar, A.; Chandler, D. Hydrogen-Bond Kinetics in Liquid Water. Nature 1996, 379, 55-57. 
36) Skarmoutsos, I.; Guardia, E. Effect of the Local Hydrogen Bonding Network on the Reorientational and Translational Dynamics in Supercritical Water. J. Chem. Phys. 2010, 132, 074502.

37) Skarmoutsos, I.; Dellis, D.; Samios, J. The Effect of Intermolecular Interactions on Local Density Inhomogeneities and Related Dynamics in Pure Supercritical Fluids. A Comparative Molecular Dynamics Simulation Study. J. Phys. Chem. B 2009, 113, 2783-2793.

38) Lee, H. S.; Tuckerman, M. E. Structure of Liquid Water at Ambient Temperature from Ab Initio Molecular Dynamics Performed in the Complete Basis Set Limit. $J$. Chem. Phys. 2006, 125, 154507.

39) Kuo, I. F.; Mundy, C. J. An ab Initio Molecular Dynamics Study of the Aqueous Liquid-Vapor Interface. Science 2004, 303, 658-660.

40) Guardia, E.; Laria, D.; Marti, J. Hydrogen Bond Structure and Dynamics in Aqueous Electrolytes at Ambient and Supercritical Conditions. J. Phys. Chem. B 2006, 110, 6332-6338.

41) Saiz, L.; Padro, J. A.; Guardia, E. Dynamics and Hydrogen Bonding in Liquid Ethanol. Mol. Phys. 1999, 97, 897-905.

42) Skarmoutsos, I.; Guardia, E. Local Structural Effects and Related Dynamics in Supercritical Ethanol. 2. Hydrogen-Bonding and Its Effect on Single Reorientational Dynamics. J. Phys. Chem. B 2009, 113, 8898-8910.

43) Skarmoutsos, I.; Guardia, E.; Samios, J. Hydrogen Bond, Electron DonorAcceptor Dimer and Residence Dynamics in Supercritical CO2-Ethanol Mixtures and the Effect of Hydrogen Bonding on Single Reorientational and Translational Dynamics: A Molecular Dynamics Simulation Study. J. Chem. Phys. 2010, 133, 014504. 
44) Soper, A.; Bruni, F.; Ricci, M. A. Site-Site Pair Correlation Functions of Water from 25 to $400{ }^{\circ} \mathrm{C}$ : Revised Analysis of New and Old Diffraction Data. J. Chem. Phys. 1997, 106, 247-254.

45) Errington, J. R.; Debenedetti, P. G. Relationship between Structural Order and the Anomalies of Liquid Water. Nature 2001, 409, 318-321.

46) DiStasio, R. A., Jr; Santra, B.; Li, Z.; Wu, X.; Car, R. The Individual and Collective Effects of Exact Exchange and Dispersion Interactions on the Ab Initio Structure of Liquid Water. J. Chem. Phys. 2014, 141, 084502.

47) Kumar, P.; Buldyrev, S. V.; Stanley, H. E. A Tetrahedral Entropy for Water. Proc. Natl. Acad. Sci. USA 2009, 106, $22130-22134$.

48) Rapaport, D. C. Hydrogen Bonds in Water: Network Organization and Lifetimes. Mol. Phys. 1983, 50, 1151-1162.

49) Chandra, A. Effects of Ion Atmosphere on Hydrogen-Bond Dynamics in Aqueous Electrolyte Solutions. Phys. Rev. Lett. 2000, 85, 768-771.

50) Matsumoto, M.; Gubbins, K. E. Hydrogen Bonding in Liquid Methanol. J. Chem. Phys. 1990, 93, 1981-1994.

51) Starr, F. W.; Nielsen, J. K.; Stanley, H. E. Hydrogen-Bond Dynamics for the Extended Simple Point-Charge Model of Water. Phys. Rev. E 2000, 62, 579-587.

52) Ando, K.; Hynes, J. T. HCl Acid Ionization in Water: A Theoretical Molecular Modeling. J. Mol. Liq. 1995, 64, 25-37.

53) Ando, K.; Hynes, J. T. Acid-Base Proton Transfer and Ion Pair Formation in Solution. Adv. Chem. Phys. 1999, 110, 381-430.

54) Marx, D.; Tuckerman, M. E.; Hutter, J.; Parrinello, M. The Nature of the Hydrated Excess Proton in Water. Nature 1999, 397, 601-604.

55) Agmon, N. The Grotthuss Mechanism. Chem. Phys. Lett. 1995, 244, 456-462. 
56) Bagchi, B. Water Dynamics in the Hydration Layer around Proteins and Micelles. Chem. Rev. 2005, 105, 3197-3219.

57) Chen, S. H.; Teixeira, J. Structure and Dynamics of Low-Temperature Water as Studied by Scattering Techniques. Adv. Chem. Phys. 1986, 64, 1-45.

58) Woutersen, S.; Emmerichs, U.; Bakker, H. J. Femtosecond Mid-IR Pump-Probe Spectroscopy of Liquid Water: Evidence of a Two-Component Structure. Science 1997, 278, 658-660.

59) Lawrence, C. P.; Skinner, J. L. Vibrational Spectroscopy of HOD in liquid $\mathrm{D}_{2} \mathrm{O}$. III. Spectral Diffusion, and Hydrogen-Bonding and Rotational Dynamics. J. Chem. Phys. 2003, 118, 264-272.

60) Tan, H. S.; Piletic, I. R.; Fayer, M. D. Orientational Dynamics of Water Confined on a Nanometer Length Scale in Reverse Micelles. J. Chem. Phys. 2005, 122, 174501. 61) Fecko, C. J.; Loparo, J. J.; Roberts, S. T.; Tokmakoff, A Local Hydrogen Bonding Dynamics and Collective Reorganization in Water: Ultrafast Infrared Spectroscopy of HOD/D 2 O. J. Chem. Phys. 2005, 122, 054506.

62) Rezus, Y. L. A.; Bakker, H. J. On the Orientational Relaxation of HDO in Liquid Water. J. Chem. Phys. 2005, 123, 114502.

63) Winkler, J.; Lindner, J.; Bürsing, H.; Vöhringer, P. Ultrafast Raman-Induced Kerr-Effect of Water: Single Molecule versus Collective Motions. J. Chem. Phys. 2000, 113, 4674-4682.

64) Stirnemann, G.; Laage, D. Direct Evidence of Angular Jumps During Water Reorientation Through Two-Dimensional Infrared Anisotropy. J. Phys. Chem. Lett. 2010, 1, 1511-1516. 
65) Chang, S. L.; Wu, T. -M.; Mou, C. -Y. Instantaneous Normal Mode Analysis of Orientational Motions in Liquid Water: Local Structural Effects. J. Chem. Phys. 2004, 121, 3605-3612.

66) Laage, D.; Hynes, J. T. A Molecular Jump Mechanism of Water Reorientation. Science 2006, 311, 832-835.

67) Laage, D.; Hynes, J. T. Do More Strongly Hydrogen-Bonded Water Molecules Reorient More Slowly? Chem. Phys. Lett. 2006, 433, 80-85.

68) Laage, D.; Hynes, J. T. On the Molecular Mechanism of Water Reorientation. $J$. Phys. Chem. B 2008, 112, 14230-14242.

69) Stirnemann, G.; Laage, D. On the Origin of the Non-Arrhenius Behavior in Water Reorientation Dynamics. J. Chem. Phys. 2012, 137, 031101.

70) Moilanen, D. E.; Fenn, E. E.; Lin, Y.-S.; Skinner, J. L.; Bagchi, B.; Fayer, M. D. Water Inertial Reorientation: Hydrogen Bond Strength and the Angular Potential. Proc. Natl. Acad. Sci. 2008, 105, 5295-5300.

71) Walrafen, G.E. in Water: A Comprehensive Treatise; Ed. F. Franks, Plenum: New York, 1972, Vol. 1, Chapt. 5

72) Downing, H. D.; Williams, D. J. Optical Constants of Water in the Infrared. $J$. Geophys. Res. 1975, 80, 1656-1661.

73) Bertie, J. E.; Ahmed, M. K.; Eysel, H. E. Infrared Intensities of Liquids. 5. Optical and Dielectric Constants, Integrated Intensities and Dipole Moment Derivatives of $\mathrm{H}_{2} \mathrm{O}$ and $\mathrm{D}_{2} \mathrm{O}$ at $22{ }^{\circ}$ C. J. Phys. Chem. 1989, 93, 2210-2218.

74) Segre, E. Nuove Bande Raman Dell’ acqua. Rend. Lincei 1931, 13, 929-938.

75) Bolla, G. Sulla Forma della Banda Raman Dell’acqua. I Il Nuovo Cimento 1932, 9, 290-298. 
76) Mallik, B.S.; Semparithi, A.; Chandra, A. Vibrational Spectral Diffusion and Hydrogen Bond Dynamics in Heavy Water from First Principles. J. Phys. Chem. A 2008, 112, 5104-5112.

77) Galvin, M.; Zerulla, D. The Extreme Low-Frequency Raman Spectrum of Liquid Water. Chem. Phys. Chem. 2011, 12, 913-914.

78) Tsai, K.H.; Ten-Ming, W. Local Structural Effects on Low-Frequency Vibrational Spectrum of Liquid Water: The Instantaneous-Normal-Mode Analysis. Chem. Phys. Lett. 2006, 417, 389-394.

79) Padro, J. A.; Marti, J. An Interpretation of the Low-Frequency Spectrum of Liquid Water. J. Chem. Phys. 2003, 118, 452-453.

80) De Santis, A.; Ercoli, A.; Rocca, D. Comment on "An Interpretation of the LowFrequency Spectrum of Liquid Water”. J. Chem. Phys. 2004, 120, 1657-1658.

81) Padro, J. A.; Marti, J. Response to "Comment on 'An Interpretation of the LowFrequency Spectrum of Liquid Water'”. J. Chem. Phys. 2004, 120, 1659-1660.

82) Sutmann, G.; Vallauri, R. Hydrogen Bonded Clusters in the Liquid Phase: I. Analysis of the Velocity Correlation Function of Water Triplets. J. Phys. Condens. Matter 1998, 10, 9231-9240.

83) Walrafen, G. E.; Fisher, M. R.; Hokmabadi, M. S.; Yang, W.-H. Temperature dependence of the low- and high-frequency Raman scattering from liquid water. $J$. Chem. Phys. 1986, 85, 6970-6982. 
Table 1: Fractions of water molecules forming $n=0-5$ hydrogen bonds and intermittent lifetimes of the corresponding HB states. Also, the HB continuous and intermittent lifetimes, reorientational correlation times and the relaxation time of the orientational tetrahedral order parameter are presented.

\begin{tabular}{lll}
\hline$n_{H B}$ & $f_{n}(\%)^{a}$ & $\tau_{H B}^{n, I}(\mathrm{fs})$ \\
\hline 0 & 0.13 & 33 \\
1 & 1.42 & 79 \\
2 & 10.56 & 192 \\
3 & 31.09 & 149 \\
4 & 52.14 & 240 \\
5 & 4.62 & 170 \\
\hline \multicolumn{3}{c}{ Average relaxation times (ps) } \\
\hline$\tau_{H B}^{C}$ & 0.46 \\
$\tau_{H B}^{I}$ & 4.9 \\
$\tau_{1 R}$ & 4.8 \\
$\tau_{2 R}$ & 2.0 \\
$\tau_{3 R}$ & 1.0 \\
$\tau_{q}$ & 0.40 \\
\hline *a A very small fraction (0.04 \% ) of water molecules forminging 6 \\
hydrogen bonds was also found
\end{tabular}


Table 2: Dependence of the libration, bending and stretching spectral peak position on the initial HB state.

\begin{tabular}{llll}
\hline$n_{H B}$ & Libration $\left(\mathrm{cm}^{-1}\right)$ & Bending $\left(\mathrm{cm}^{-1}\right)$ & Stretching $\left(\mathrm{cm}^{-1}\right)$ \\
\hline 0 & 360 & 1574 & 3406 \\
1 & 395 & 1583 & 3411 \\
2 & 480 & 1581 & 3343 \\
3 & 514 & 1585 & 3329 \\
4 & 536 & 1585 & 3291 \\
5 & 559 & 1578 & 3250 \\
\hline
\end{tabular}



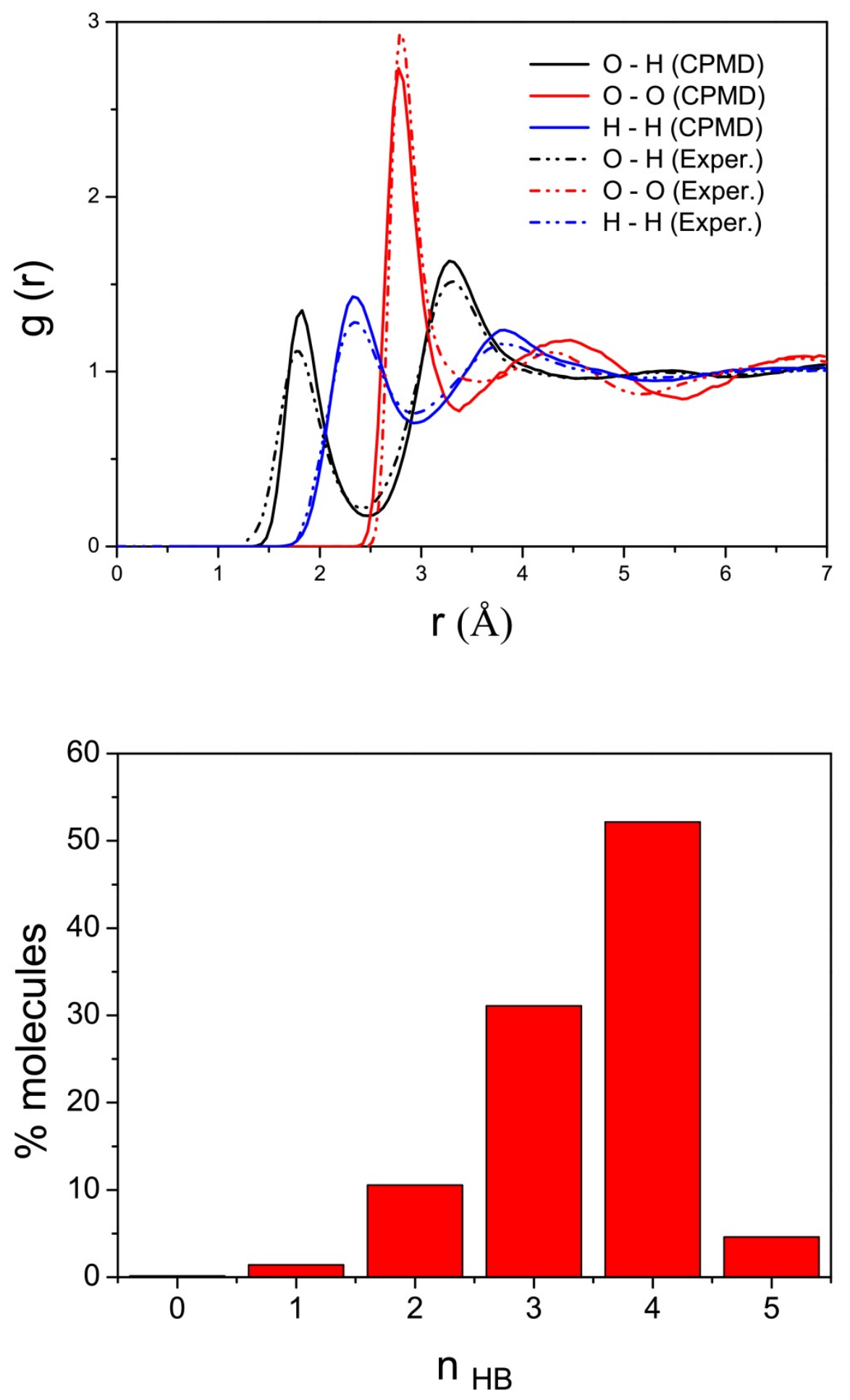

Figure 1 

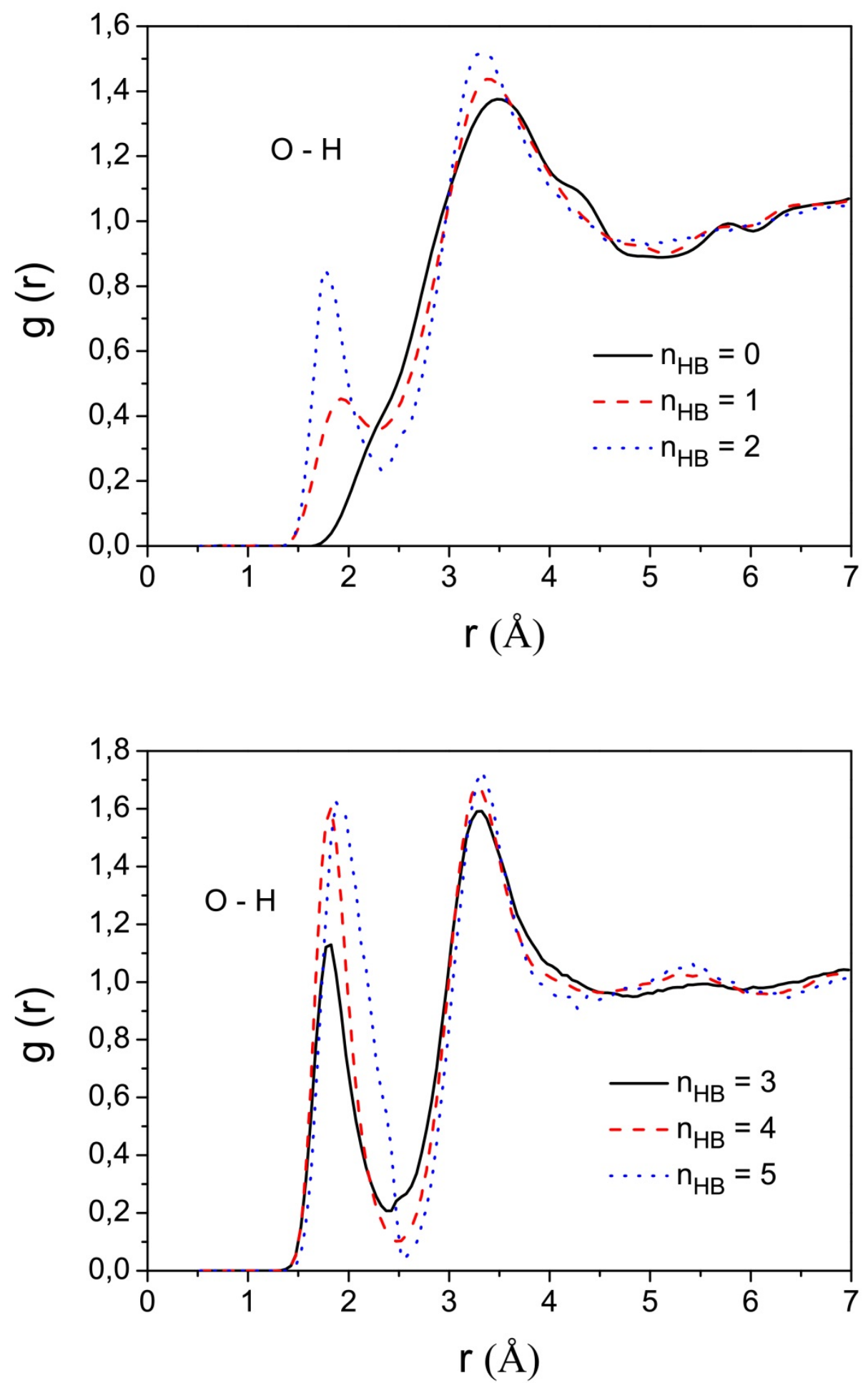

Figure 2 

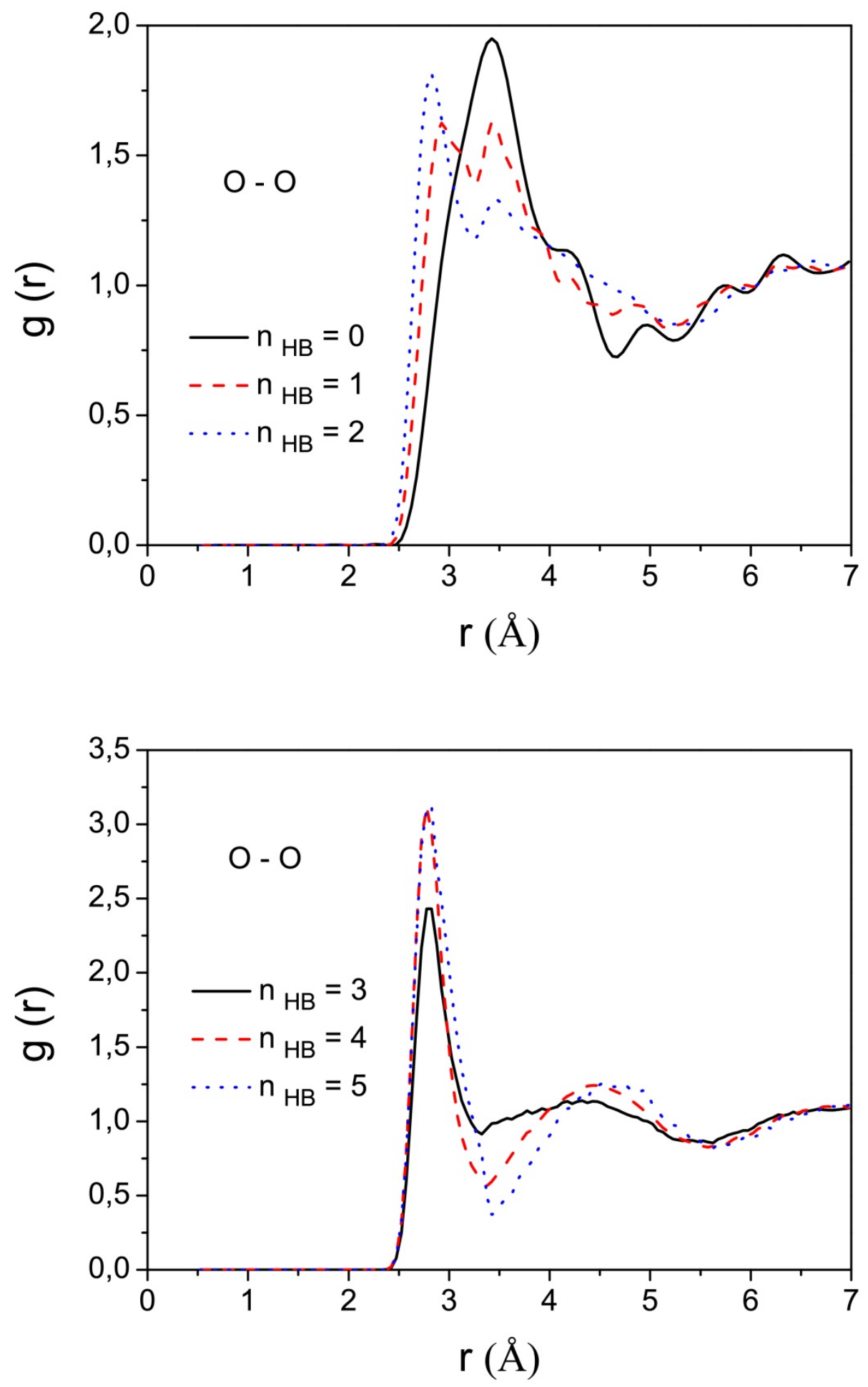

Figure 3 

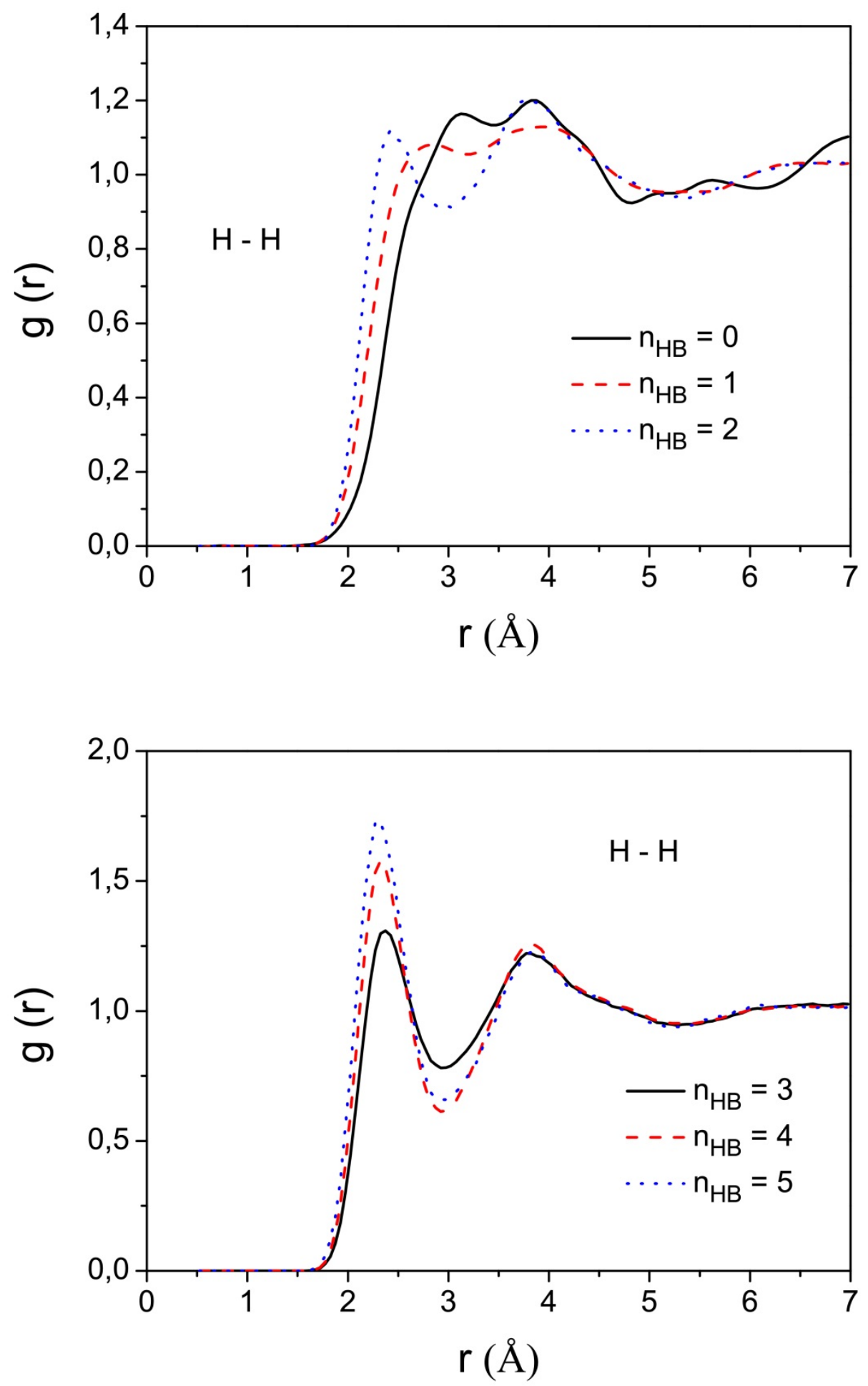

Figure 4 


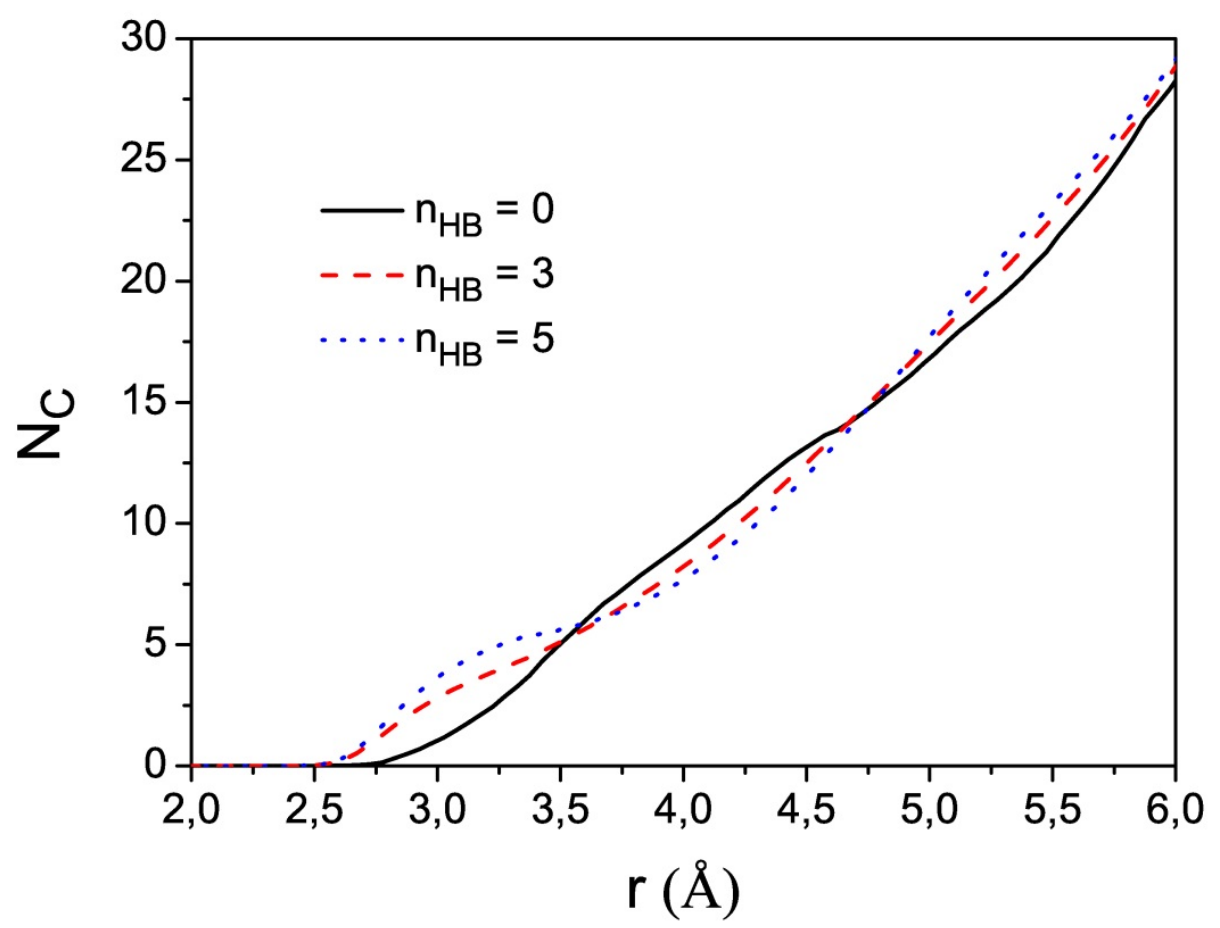

Figure 5 

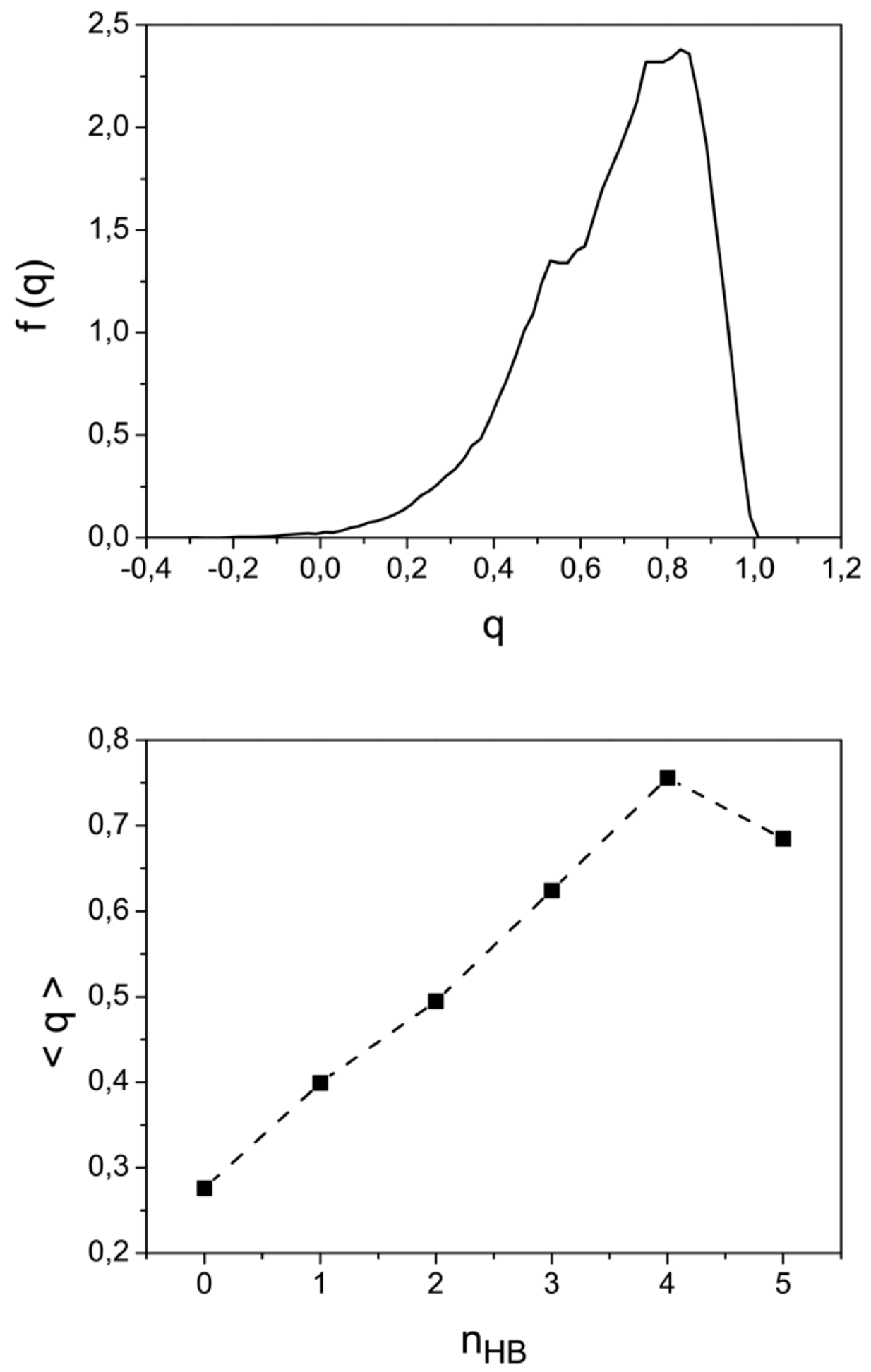

Figure 6 

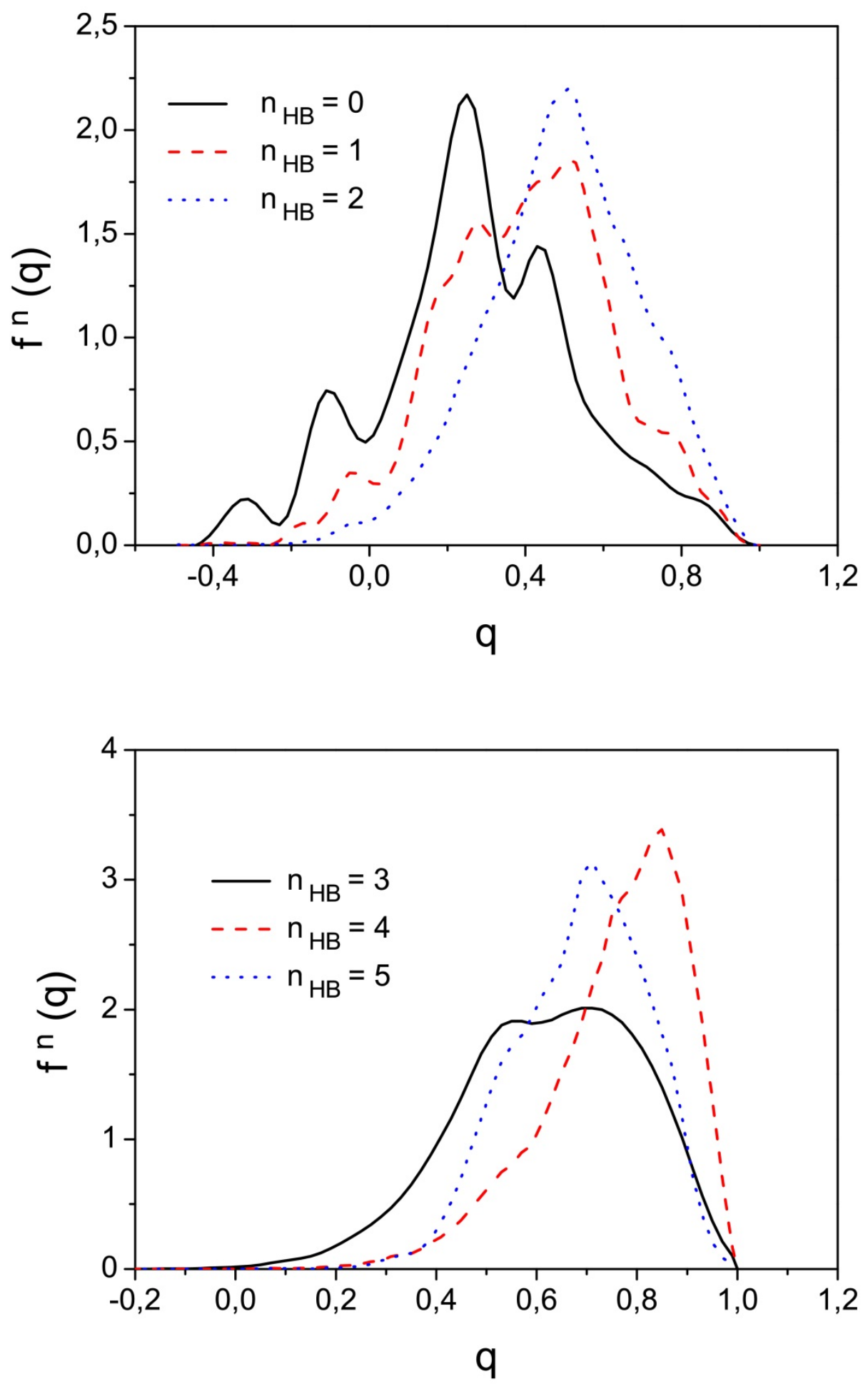

Figure 7 


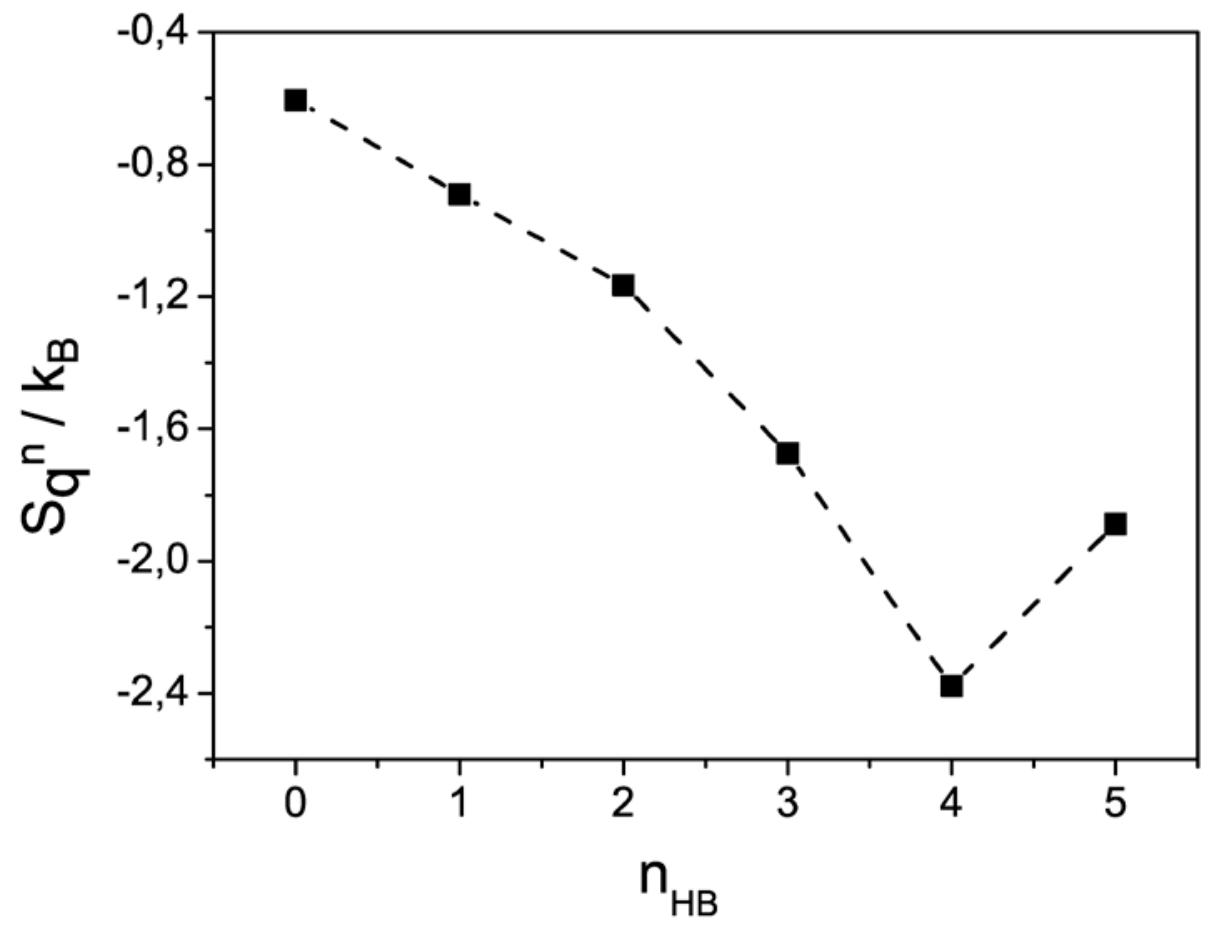

Figure 8 

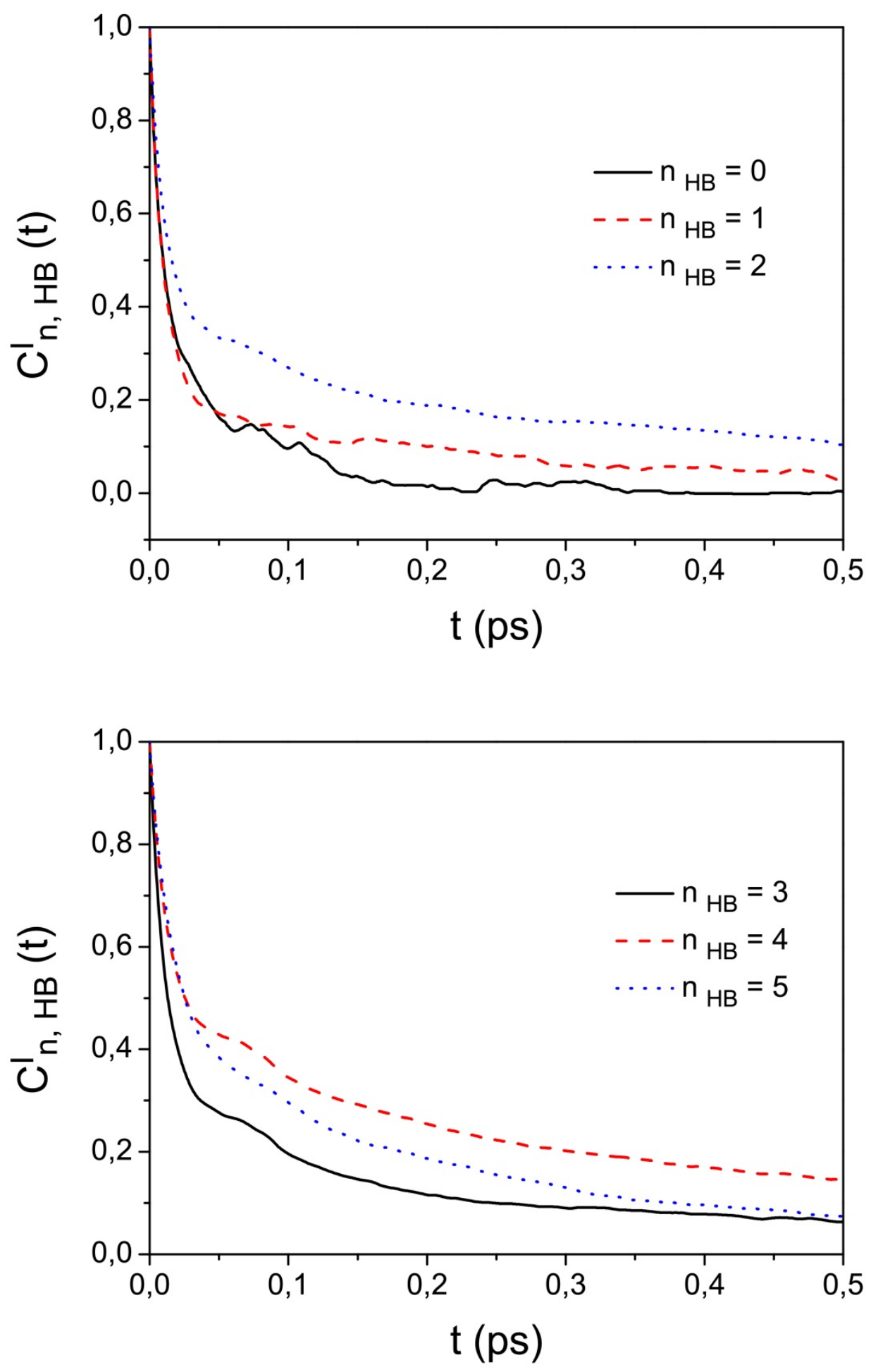

Figure 9 

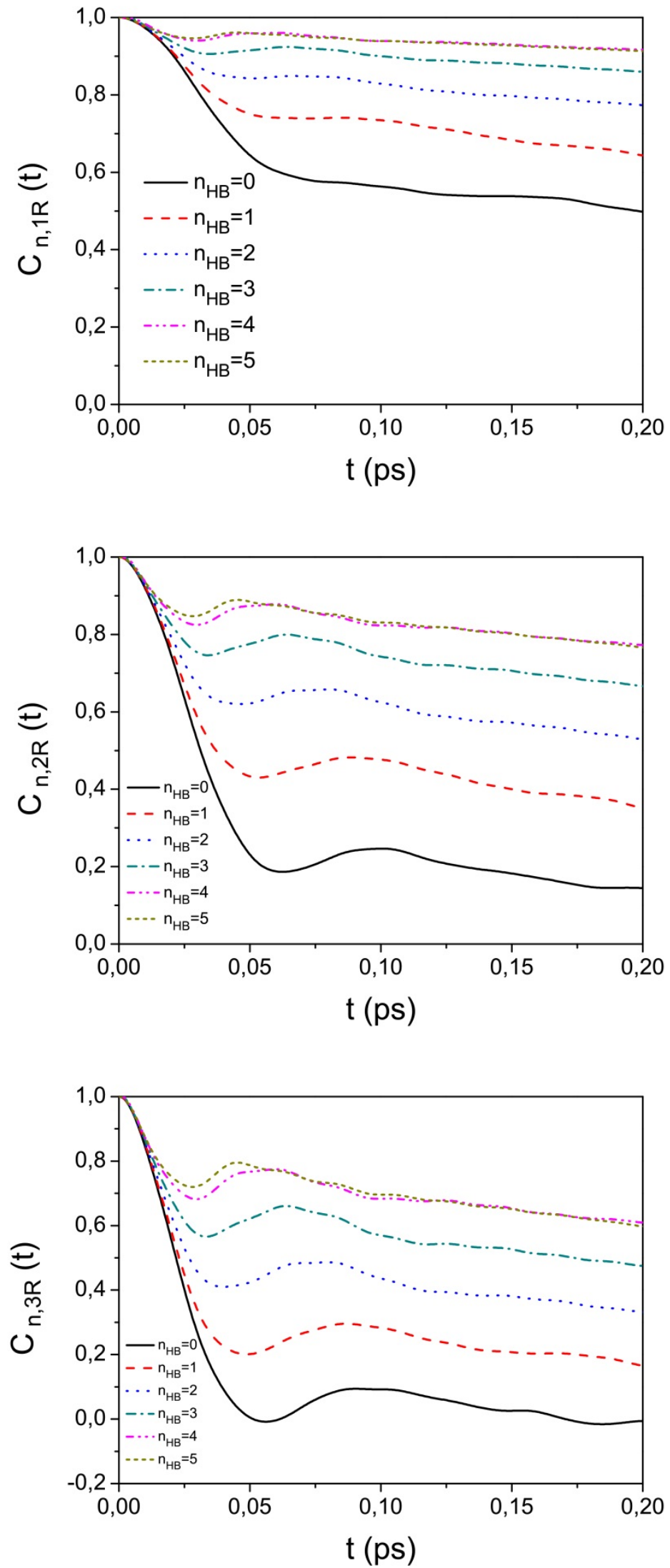

Figure 10 

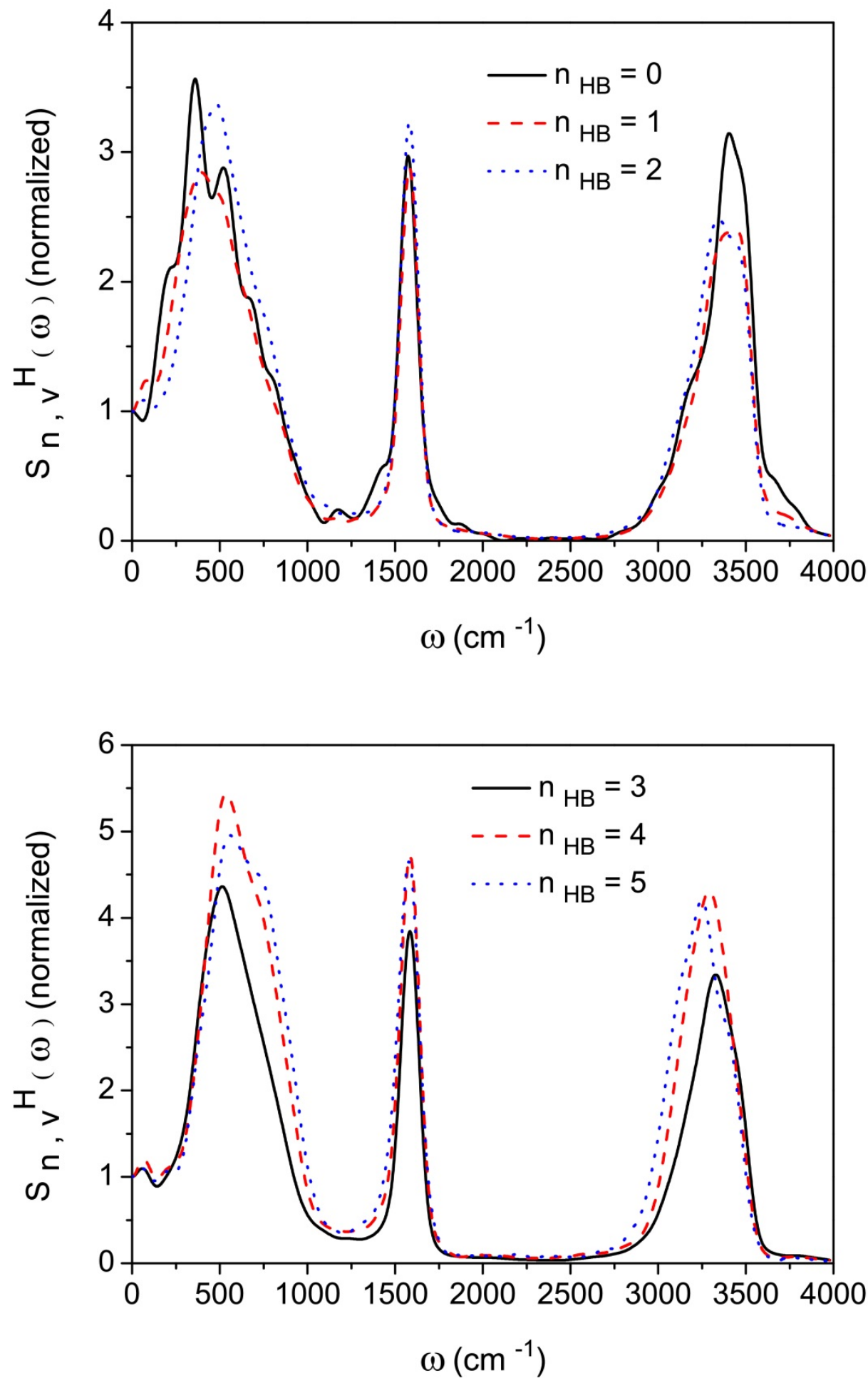

Figure 11 

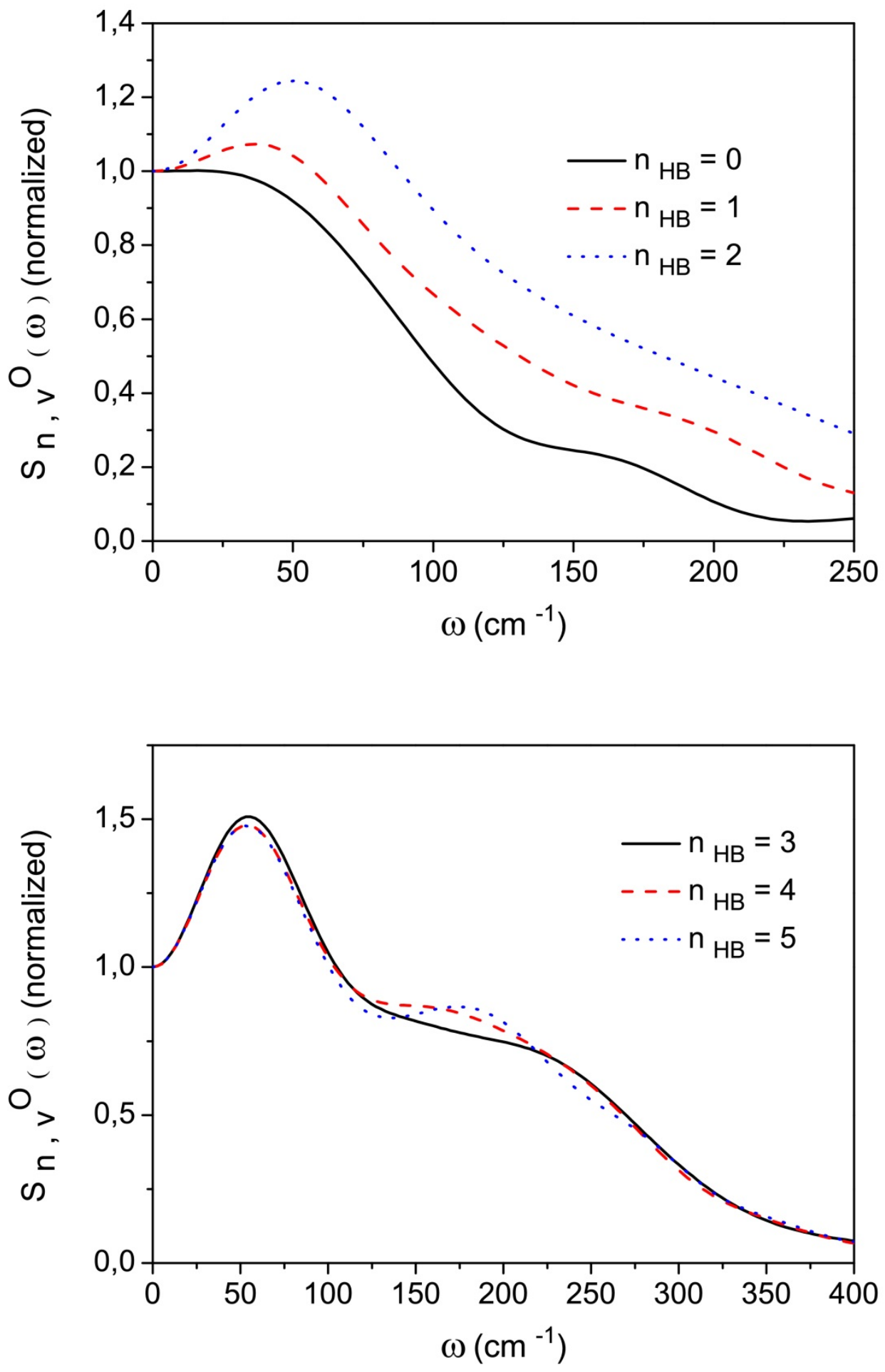

Figure 12 


\section{Figure Captions}

Figure 1: (Top) Atom-atom pair radial distribution functions. Solid line: simulated results; dashed line: experimental results (see Ref. 28. (Bottom) Percentages of molecules with $\mathrm{n} \mathrm{H}$ Bonds.

Figure 2: O-O pair radial distribution functions $g^{n}$ oo $(r)$ for different HB states.

Figure 3: $\mathrm{O}-\mathrm{H}$ pair radial distribution functions $g^{n} \mathrm{OH}(r)$ for different $\mathrm{HB}$ states.

Figure 4: H-H pair radial distribution functions $g^{n}{ }_{H H}(r)$ for different HB states.

Figure 5: Coordination numbers as a function of the distance around molecules for different HB states.

Figure 6: (Top) Probability distribution function $f(q)$ of the tetrahedral order parameter. (Bottom) Average tetrahedral order parameter for different HB states.

Figure 7: Probability distribution function $f^{n}(q)$ of the tetrahedral order parameter for different HB classes.

Figure 8: Tetrahedral entropy contributions from different HB states.

Figure 9: Intermittent $\mathrm{HB}$ time correlation functions functions $C_{n, H B}^{I}(t)$ for different HB states.

Figure 10: $\mathrm{O}-\mathrm{H}$ reorientational time correlation functions $C_{n, \ell R}(t)$ for different $\mathrm{HB}$ states.

Figure 11: Hydrogen spectral densities $S_{n, v}{ }^{H}(\omega)$ for different HB states.

Figure 12: Oxygen spectral densities $S_{n, v}{ }^{O}(\omega)$ for different HB states. 


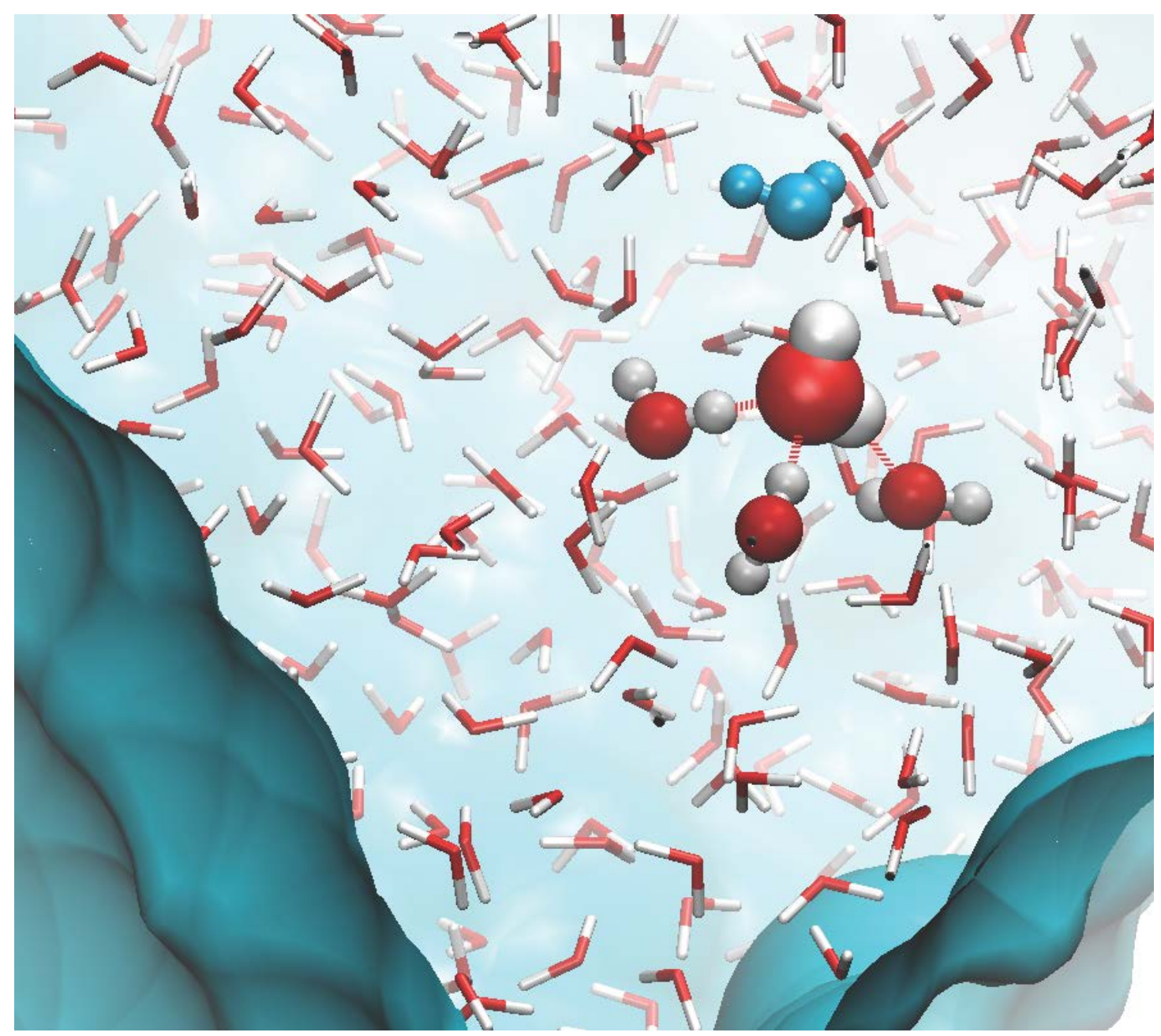

Table of Contents Graphic 